\title{
AN EXPERIMENTAL HIGH ENERGY PHYSICS PROGRAM
}

within the

DEPARTMENT OF PHYSICS

PURDUE UNIVERSITY

DOE/ER/40681--1

West Lafayette, Indiana 47907

DE92 013718

Supported by

U.S. DEPARTMENT OF ENERGY

Through

Division of Sponsored Programs

Purdue Research Foundation

West Lafayette, Indiana

\section{PROGRESS}

\author{
for \\ $11 / 1 / 9 d-10 / 31 / 92$.
}

Current Grant Number: DE-FG02-19ER40681 Task A
Principal Investigators: J. A. Gaidos F. J. Loeffler
R. L. Mcllwain,
D. H. Miller*, T. R. Palfrey E. I. Shibata, I. P. Shipsey

April 1992

*Spokesperson

\section{DISCLAIMER}

This report was prepared as an account of work sponsored by an agency of the United States Government. Neither the United States Government nor any agency thereof, nor any of their employees, makes any warranty, express or implied, or assumes any legal liability or responsibility for the accuracy, completeness, or usefulness of any information, apparatus, product, or process disclosed, or represents that its use would not infringe privately owned rights. Reference herein to any specific commercial product, process, or service by trade name, trademark, manufacturer, or otherwise does not necessarily constitute or imply its endorsement, recommendation, or favoring by the United States Government or any agency thereof. The views and opinions of authors expressed herein do not necessarily state or reflect those of the United States Government or any agency thereof. 
TABLE OF CONTENTS

1. CLEO 3

2. Gas Microstrip Detectors 30

3. Particle Astrophysics 33

$\begin{array}{ll}\text { 4. References } & 38\end{array}$ 


\section{CHAPTER 1}

\section{CLEO}

During 1991 the Purdue group has been involved in upgrading performance of the CLEOII detector to match the performance of CESR which has given us the world's largst sample of B mesons. Physics analyses based on 1 million B meson pairs requires greatly increased understanding of detector performance if systematic errors are not to dominate statiscal ones. Accordingly the Monte Carlo has undergone considerable refinement. In the following sections we describe the present status of the various detector sub-systems including upgrades. We concentrate mainly on changes and improvements achieved within the past year, especially those where Purdue has played a major part.

\subsection{DETECTOR.}

The CLEO-II detector is shown schematically in Fig. 1. From the interaction point radially outward, it is made up of a tracking system (which also measures $\mathrm{dE} / \mathrm{dx}$ ), a time-of-flight system, an electromagnetic calorimeter, and a muon identification system. A description of the detector is given in CLNS $91 / 1122$, which has been submitted to NIM. In addition, a description of the trigger to which Shibata, McIlwain and students made a major contribution has been published in NIM in $1991^{2}$ and a description of the muon detector for which Shipsey played a leading role has been accepted by NIM and is in press ${ }^{3}$

\subsubsection{Tracking.}

The tracking devices in CLEO II consist of a main drift chamber (DR) of $1-\mathrm{m}$ radius, surrounding two vertex drift chambers which extend to within $4 \mathrm{~cm}$ of the interaction point. When we installed the larger CLEO II superconducting coil, we also increased the magnetic field from $1 \mathrm{~T}$ to $1.5 \mathrm{~T}$ to give better charged particle curvature measurements. Much effort has gone into realizing that improvement and Purdue post-doc Schaffner has play a key role in this. Naively, we would anticipate an improvement in momentum resolution of about a factor of 1.5 at high momenta, due to the increased magnetic field. In fact, somewhat less than this will be achieved, since the spatial resolution and efficiency are degraded substantially by magnetic field effects near the edges of the DR cells. Figure 2 shows a fit to $e^{+} e^{-} \rightarrow \mu^{+} \mu^{-}$ data taken at the $4 \mathrm{~S}$ resonance. The resolution is $57 \mathrm{MeV} / \mathrm{c}$, a factor of 1.47 times better than the typical CLEO 1.5 resolution of $83 \mathrm{MeV} / \mathrm{c}$.

The higher magnetic field of CLEO II requires improved handling of "curlers", tracks with insufficient transverse momentum to reach the outer radius of the DR. Any track with transverse momenta below $225 \mathrm{MeV} / \mathrm{c}$ falls into this category now, up from $150 \mathrm{MeV} / \mathrm{c}$ for CLEO 1.5. Such tracks are particularly important for the reconstruction of $D^{*+} \rightarrow D^{0} \pi^{+}$, which usually produces a pion in this momentum range (this is especially true for $D^{*+}$ 's from B's and for $\Upsilon(3 S) \rightarrow \Upsilon(2 S) \pi^{+} \pi^{-}$studied by Purdue graduate student Jay Dominick). Schaffner has improved our efficiency for such low momentum tracks by modifying several aspects of the track finding. The pattern recognition of short track segments was altered to allow segments that are less radial, and to improve the way the shorter segments are joined to make tracks. We have aloo begun to use the charge-division information available in the 


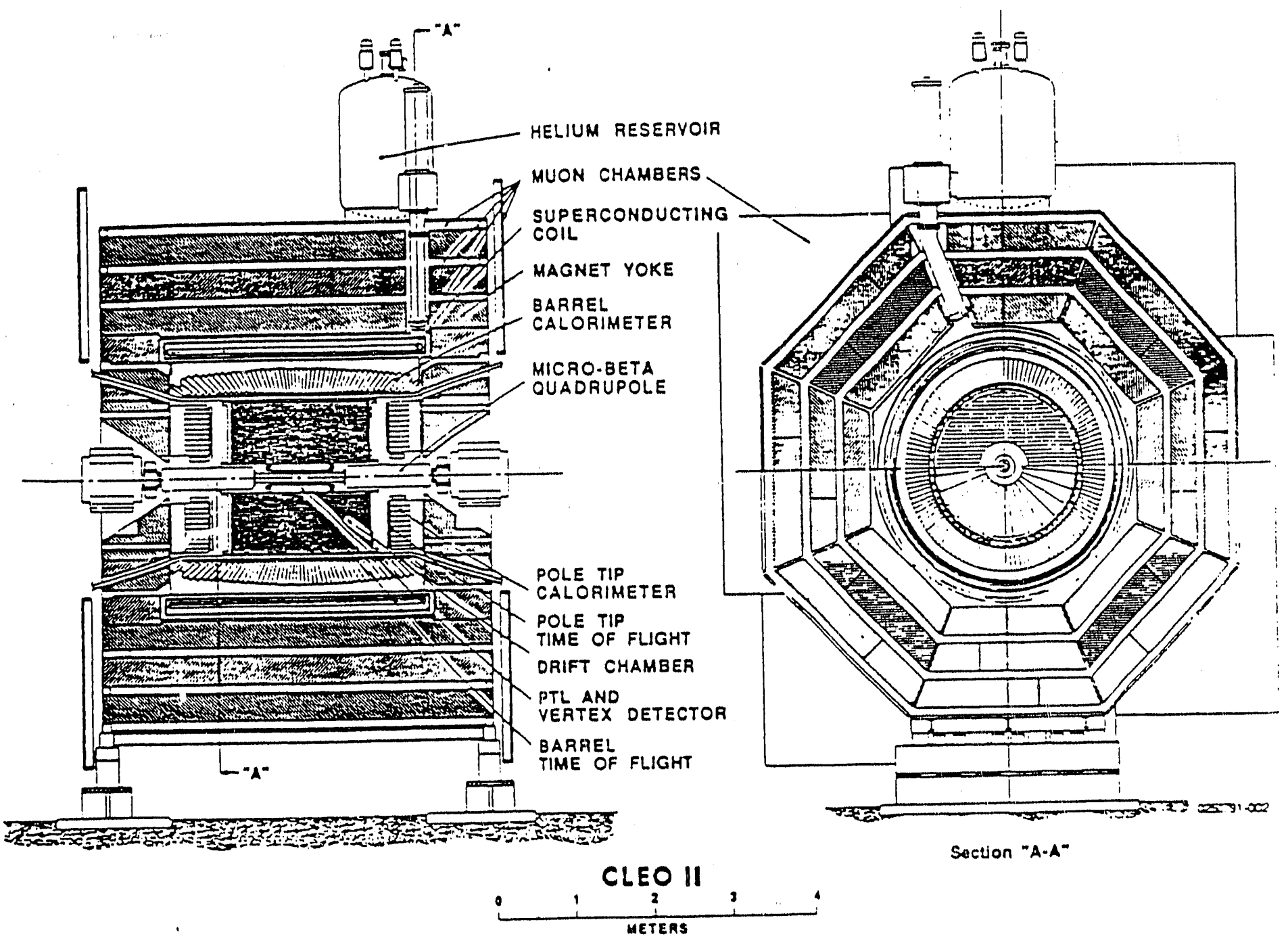

Figure 1 The CLEO-II detector. 


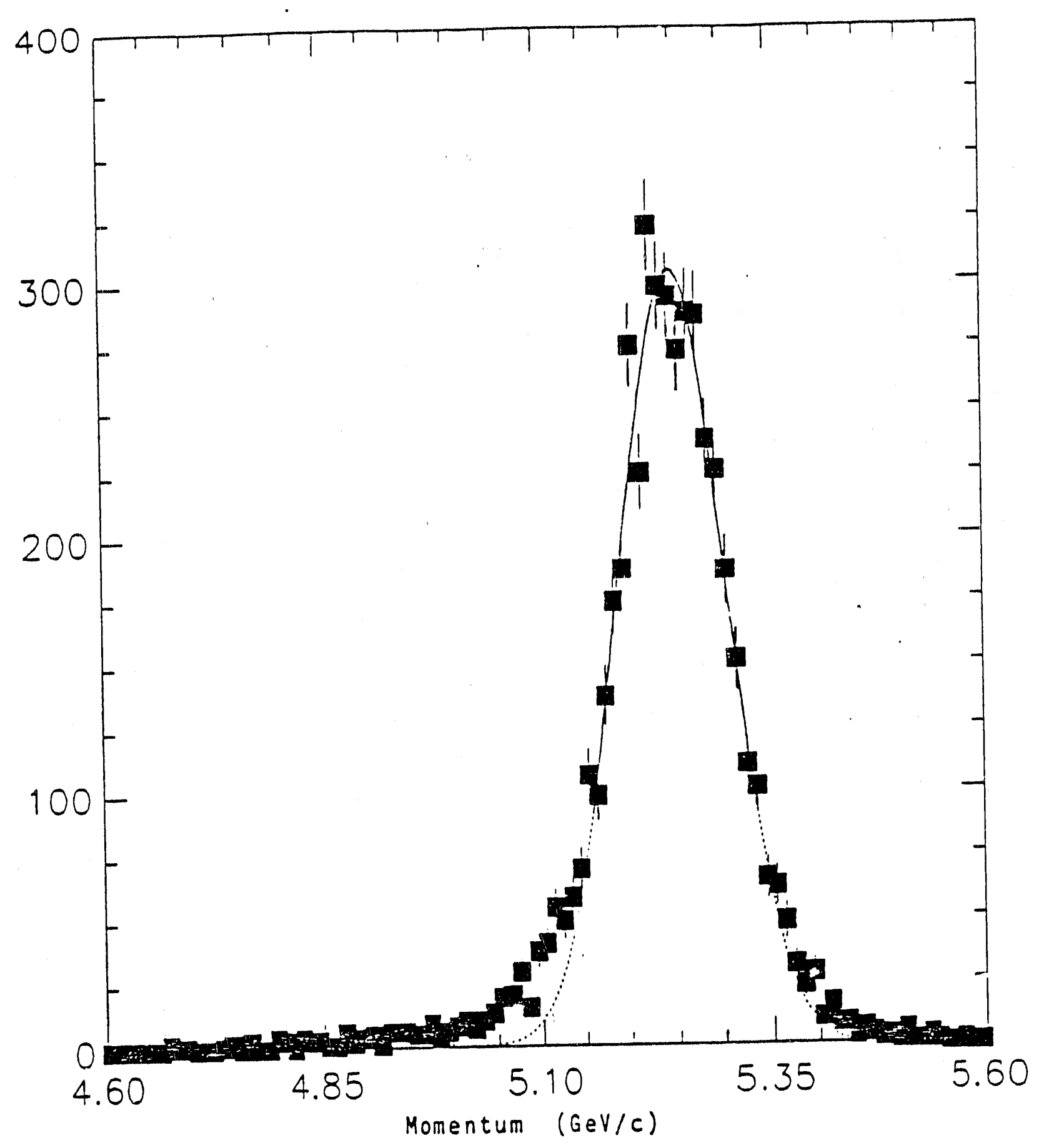

Figure 2 Momentum distribution for muons from $e^{+} e^{-} \rightarrow \mu^{+} \mu^{-}$ 
middle tracking chamber. This provides additional measurements of the $z$ position of tracks, which formerly was often inadequate for tracks that curled within the first few layers of the DR.

Other measures of performance have dramatically improved during the past year. In particular, the impact parameter resolution for high-momentum tracks is now close to the $80 \mu \mathrm{m}$ achieved with CLEO 1.5. This required detailed understanding of the properties of the innermost straw-tube vertex detector. Further improvements, to perhaps $50 \mu \mathrm{m}$ or better, are expected from a recent change in drift gas from argon-ethane to dimethyl ether (DME) in this chamber. This will greatly improve our capability for lifetime physics (especially $\tau$ ) and opens the possibility of tagging $\tau$ and charm decays by the separation of their decay vertices from the event origin.

\section{$1.1 .2 \mathrm{dE} / \mathrm{dx}$.}

Specific ionization is measured in the 51 layers of the drift chamber to help identify particles. To obtain optimal resolution, the data must be corrected for dip angle saturation, drift distance, entrance engle, and proximity to the stereo layers. Purdue students Brown, Dominick and Modesitt have worked to correct the data using a five-dimensional mapping. The system performs marginally better than CLEO 1.5. For long tracks, resolution for Bhabha electrons is $6.2 \%$. The $\mathrm{dE} / \mathrm{dx}$ system provides $2 \sigma \pi / \mathrm{K}$ separation up to $0.7 \mathrm{GeV} / \mathrm{c}$, as shown in Fig. 3.

\subsubsection{Time of Flight.}

The time-of-flight counters cover $96 \%$ of $4 \pi$. The barrel scintillators show a timing resolution of $139 \mathrm{ps}$ for Bhabha events. In the higher multiplicities of hadronic events, the design resolution of $150 \mathrm{ps}$ is achieved. This provides $2 \sigma \pi / \mathrm{K}$ separation up to $1.1 \mathrm{GeV} / \mathrm{c}$ and $2 \sigma \mathrm{p} / \mathrm{K}$ separation up to $1.7 \mathrm{GeV} / \mathrm{c}$. The endcap time-of-flight counters are viewed from only one end, and function in a less optimal environment. To date, resolutions of $240 \mathrm{ps}$ in Bhabha events and $400 \mathrm{ps}$ in hadronic events have been observed. This separates pions and kaons up to $0.6 \mathrm{GeV} / \mathrm{c}$, and can provide redundancy for the $\mathrm{dE} / \mathrm{dx}$ system in the forward regions where the $\mathrm{dE} / \mathrm{dx}$ resolution is poorest. To obtain global particle identication Purdue student David Brown has combined the time-of-flight information with $\mathrm{dE} / \mathrm{dx}$ information, his code is now a standard part of the CLEO analysis package.

\subsubsection{Electromagnetic Calorimeter.}

The CLEO II calorimeter, composed of 7800 CsI crystals with photodiode readout inside the solenoid coil, continues to perform very well. The calibration of electronics, relative crystal-to-crystal gains, and absolute energy scale have successfully maintained excellent resolution and stability. In the barrel region energy resolution is $3 \%$ at $150 \mathrm{MeV}$ and $1.5 \%$ at $5 \mathrm{GeV}$. Purdue Post Doc Bortoletto has been involved in crystal calibration.

Electron identification, which depends on the shower energy to track momentum ratio as well as the specific ionization measurement in the main drift chamber, has continued to underso improvements. Hadron rejection of several hundred to one is achieved with efficiency exceeding $90 \%$. Physics analyses routinely use calorimeter information for photons and electrons. 


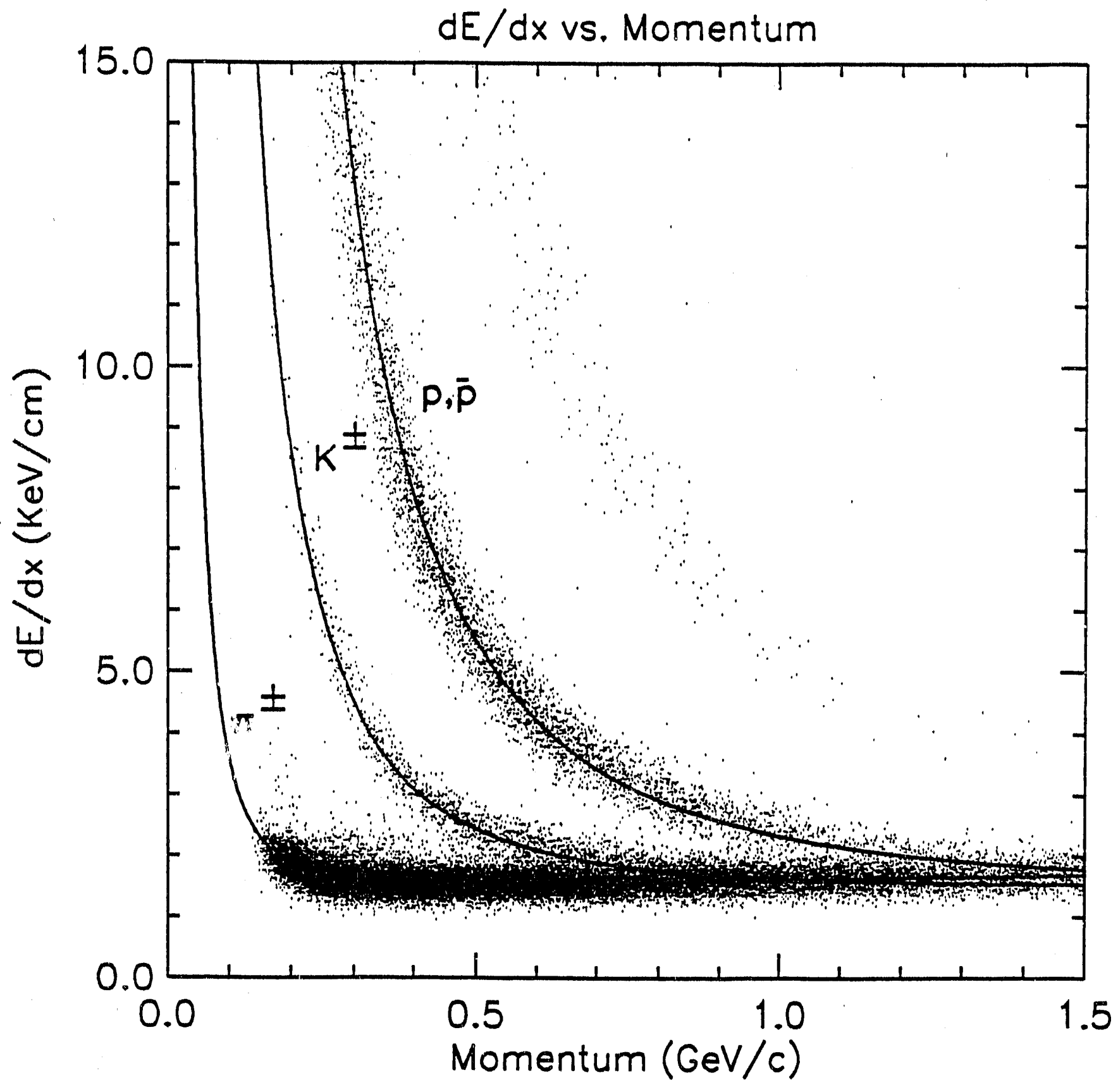

Figure 3 Drift Chamber $d E / d x$. 
There have been two very encouraging signs for the longevity of the calorimeter. First, there is no evidence for radiation damage in the endcap crystals lying in the horizontal plane at the $5 \%$ level in light emission, despite exposures of $100 \mathrm{Rad}$. Second, the inaccessible diodes and preamplifiers, four of which are mounted directly to each crystal and read out independently for redundancy, have experienced a very low failure rate. A total of 169 channels out of 31200 have been deactivated, and the rate has stabilized to approximately 25 per year. Only one crystal has two of its four diodes/preamp combinations deactivated.

There is only one aspect of the calorimeter behavior of concern. The Bhabha calibration of crystal-to-crystal gains shows a clear trend for significant loss of light output in some crystals over time; this has forced us to recalibrate the crystals every 10 weeks or so. About $10 \%$ of all crystals changed more than $3 \%$ in light output in 21 months; about $1 \%$ changed more than $3 \%$ in 2 months. These crystals are not preferentially located in any particular region of the detector and do not show any electronic anomalies. At present we have no explanation for this behavior.

\subsubsection{Muon Identification.}

The muon identification system has been operating smoothly and without trouble. The system hardware, its construction, testing and performance, and the associated analysis and simulation software are described in a NIM paper in press. This detector provides greater solid angle coverage and lower fake rates than the detctor it replaced. Shipsey played a major role in the construction of this detector.

\subsubsection{Trigger and Data Acquisition System.}

During the summer 1991 shutdown, a new data acquisition system was installed in CLEO. In the new system the crate readout is done in parallel using a faster network, resulting in an order of magnitude reduction in event readout time, to about $2 \mathrm{msec}$. (An additional factor of two improvement is possible with a modest amouni of effort). This network is interfaced to VME where 68040 processors sparsify the data and put it in ZEBRA format. Events are then subjected to an additional level of filtering in software ("LEVEL3") before being written to a disk. The expected rate caparity is 50 events/sec. Currently, we are limited to 35 events per second by the sparsification time, but this still represents a factor of five improvement over the previous system. The live time, typically around $94 \%$, is also significantly better than in the previous system.

The increased rate capacity has allowed a significantly looser trigger than in CLEO 1.5 providing greater redundancy for hadronic and Bhabha events and improved acceptance for low multiplicity physics such as that of tau pairs, two photon interactions. Typical trigger rates at $2 \times 10^{32} / \mathrm{s}-\mathrm{cm}^{2}$ are $15-20 \mathrm{KHz}$ for Level $0,30-40 \mathrm{~Hz}$ for Level $1,15-20 \mathrm{~Hz}$ for Level 2 , and $8-12 \mathrm{~Hz}$ written to tape. Even looser triggers are being investigated.

\subsubsection{Software and Computing.}

During the past year most of the CLEO software twas settled down into a reasonably well organized state. New library procedures have been developed which allow any CLEO library and all its compilation directives to be kept in a single ASCII source code file. This can be copied to any of the five current CLEO platforms (VAX/VMS, DECstation, Silicon Graphics, IBM RS/6000, or HP-PA 9000/700) and there used to build an np-to-date library 
without human intervention. This has greatly eased the problem of maintaining code in all machines, both at Cornell and at Purdue.

Data reduction is running relatively smoothly both at Cornell and Florida, DECstation 5000 's process about $120 \mathrm{pb}^{-1}$ per week. The CLEO-II data (1600 $\mathrm{pb}^{-1}$ and growing) is currently being reprocessed, with improved tracking programs and calibrations. We expect to catch up with the data by the end of May.

CABS (CLEO Analysis By Script) is a software innovation introduced in the past year. A physicist can describe particles, cuts, and decay chains in a script, and this script is translated by a $\mathrm{C}++$ program into a normal CLEO data analysis executable. This avoids the need for physicists to write the complicated FORTRAN loop structures which so often give rise to errcrs. For example one Purdue student, Mike Modesitt, is seeking all B's which decay into a $D_{s}$ and $D^{*}$, where both charmed particles decay in any of a dozen modes. CABS is now searching for fully reconstructed $B$ tags for mixing and the measurement of the $B^{-}$ and $\overline{\mathrm{B}}^{0}$ semileptonic decay rates.

Last year, Cornell bought an SGI 4D/380 to supply 120 MIPS computing power for physics data analysis which is already saturated. The central VAX, although upgraded to a 40 MIPS VAX 6000/540, remains saturated. Between them, the two machines have over 65 GByte of disk storage. This is necessary because the data sample is already 20 GBytes, and it is growing at the rate of about 1 GByte per month.

We need to increase the computing power available to physicists, but cannot afford to make a dramatic increase in the power of the central computers. To maximize available computing power, we are forming analysis centers consisting of a workstation cluster with sidecars and $4 \mathrm{~mm}$ tape drives. Purdue group members resident at Cornell will constitute one such analysis center.

\subsubsection{Silicon Vertex Detector Development}

The CLEO collaboration decided in September 1991 to install a silicon detector into CLEO II in mid-1993. This detector is expected to enhance vertex detection significantly. The expected physics payoff is discussed in the Gas Microstrip section of the proposal. The detector consists of three layers of double-sided silicon strip detectors, at radii of $2.3 \mathrm{~cm}$, $3.2 \mathrm{~cm}$, and $4.8 \mathrm{~cm}$, arranged in an approximately octagonal configuration around a $2 \mathrm{~cm}$ radius beampipe. Support structures will be made from boron carbide foam. For tracks of $3(1) \mathrm{GeV} / \mathrm{c}$ momentum, the detector will provide impact parameter resolutions of 25(45) $\mu m$ in $r \phi$ and $40(55) \mu m$ in $z$. The work is being carried out at Cornell, UCSB, Harvard and Syracuse Thirty-two full-size detectors were ordered from Hamamatsu Photonics for deliver in September 1992. A prototype water cooled beampipe is being made. Prototype hybrid boards for mechanical testing have already been received, and electrically functional boards are expected in May. Installation of the detector in CLEO is projected for May/June 1993. 


\subsection{PUBLICATIONS AND ANALYSIS}

Since January 1, 1991, 13 publications have appeared from the CLEO collaboration, 3 more have been accepted for publication, 2 have been submitted, and 4 are in an advanced stage of preparation. These are:

1. Exclusive and Inclusive Semileptonic Decays of B Mesons to D Mesons, R. Fulton et al., , Phys. Rev. D 43, 651 (1991)

2. Study of $\pi^{+} \pi^{-}$Transitions from the $\Upsilon(3 S)$ and a Search for the $h_{b}$, I. Brock et al., , Phys. Rev. D 43, 1448 (1991)

3. Study of $D^{0}$ decays into final states with a $\pi^{0}$ or $\eta$, K. Kinoshita et al., , Phys. Rev. $D$ 43, 2836 (1991)

4. Inclusive Production of the Charmed Baryon $\Lambda_{c}$ from $e^{+} e^{-}$annihiliations at $\sqrt{s}=10.55$ GeV, P. Avery et al., , Phys. Rev. D 43, 3599 (1991)

5. Study of continuum $D^{*}$ spin alignment, Y. Kubota et al., , Phys. Rev. D 44, 593 (1991)

6. Measurement of the Inclusive $B^{*}$ Cross Section above the $\Upsilon(4 \mathrm{~S})$, D. Akerib et al., , Phys. Rev. Lett. 67, 1692 (1991)

7. Inclusive $\chi(2 \mathrm{P})$ Production in $\Upsilon(3 \mathrm{~S})$ Decay, R. Morrison et al., , Phys. Rev. Lett. 67, 1696 (1991)

8. Unusual decay modes of $D^{0}$ and $\mathrm{D}^{+}$mesons, R. Ammar et al., , Phys. Rev. D 44, 3383 (1991)

9. Measurement of the ratio $\mathrm{B}\left(\mathrm{D}^{0} \rightarrow \mathrm{K}^{*-} e^{+} \nu_{e}\right) / \mathrm{B}\left(\mathrm{D}^{0} \rightarrow \mathrm{K}^{-} e^{+} \nu_{e}\right)$, G. Crawford et al., , Phys. Rev. D 44, 3394 (1991)

10. Inclusive and exclusive decays of $B$ mesons to final states including charm and charmonium mesons, D. Bortoletto et al., , Phys. Rev. D 45, 1 (1992)

11. The Decays $D_{a}^{+} \rightarrow \eta \pi^{+}$and $\eta^{\prime} \eta^{+}$, P. Avery et al., , Phys. Rev. Lett. 68, 1275 (1992)

12. The Decays $D_{s}^{+} \rightarrow \eta \rho^{+}, \eta^{\prime} \rho^{+}$and $\phi \rho^{+}$, S. Henderson et al., , Phys. Rev. Lett. 68, 1279 (1992)

13. Measurement of baryon production in B-decay, G. Crawford et al., , p D45, 752 (1992)

14. Measurement of semileptonic branching fractions at the $\Upsilon(4 \mathrm{~S})$ resonance, $S$. Henderson et al., , accepted for publication in Physical Review D.

15. The electronic branching ratio of the $\tau$ lepton, CLNS 91-1123, CLEO 91-10, accepted for publication in Physical Review D (May 1, 1992).

16. Two-body $D_{s}^{+}$decays to $\eta \pi^{+}, \eta^{\prime} \pi^{+}, \eta \rho^{+}, \eta^{\prime} \rho^{+}$, and $\phi \rho^{+}$, CLEO 91-9, accepted for publication in Physical Review D.

17. Observation of the Decay $\Xi_{c}^{0} \rightarrow \Omega^{-} \mathrm{K}$, CLEO 91-11, R. Ammar et al., , submitted for publication in Physics Letivers B.

18. Measurement of the event shapes of $\chi_{b}^{\prime}$ decays, presently in preparation.

19. Determination of $\mathrm{V}$-A dominance in B-meson decays, presently in preparation. 
20. Determination of $D^{*}$ branching ratios, presently in preparation.

21. Measurement of the lifetime of the tau lepton, presently in preparation.

There were also fourteen papers submitted to the Photon-Lepton Symposium in Geneva in July, 1991 (many of which have subsequently been turned into journal publications). These titles, and the appropriate physics topics, are:

T-physics

1. Electronic Branching Ratio of the Tau Lepton

2. A Precision Measurement of the Tau Lepton Lifetime

3. Tau Decays to Single Charged Hadrons plus $\pi^{0}$ 's

\section{Charm}

1. Decays of the $\Lambda_{0}$ to Final States with Photons

2. Study of $\mathrm{D}^{0}$ Decays to Neutral Particles

3. The Decay $\Lambda_{c} \rightarrow X \Lambda \ell \nu_{\ell}$ and a Normalization for $\Lambda_{c} \rightarrow \mathrm{pK} \pi$

4. Measurement of the $\bar{D}^{*}(2010)$ Branching Fractions

5. Two-Body $D$, Decays to $\eta \pi^{+}, \eta^{\prime} \pi^{+}, \eta \rho^{+}, \eta^{\prime} \rho^{+}$, and $\phi \rho^{+}$

6. Observation of the Decay $\Xi_{c}^{0} \rightarrow \Omega K^{+}$

\section{Bottom}

1. $\psi$ Production in $e^{+} e^{-}$Annihilations in the $\Upsilon(4 \mathrm{~S})$ Energy Region

2. Inclusive and Exclusive Decays of $B$ Mesons to $\chi_{c 1}$

3. Search for Electromagnetic-Penguin-Mediated B Decays

\section{Resonance/Fragmentation}

1. Analysis of Hadronic Transitions in Decays of the $\Upsilon(3 S)$

2. Fragmentation Studies in the $10 \mathrm{GeV}$ Energy Region

\subsubsection{CLEO-II dataset}

During 1991, the CLEO-II detector collected over $1 \mathrm{fb}^{-1}$ of data; in its present configuration with the new DAQ-90 data acquisition system, $701.5 p b^{-1}$ were collected between installation on September 1, 1991, and March 1, 1992. In the following sections we will discuss some of the exciting new results we have obtained but will concentrate mainly on those where Purdue people have been involved.

The table below summarizes our accumulated luminosity since turn-on of CLEO-II in 1989 and since January 1, 1992. Data accumulated by CLEO-I prior to 1989 is tabulated in the appendix at the end of this document. 


\begin{tabular}{|c|c|c|}
\hline Beam Energy & Run region & $\mathcal{L}\left(p b^{-1}\right)$ \\
\hline 5.177 & $\Upsilon(3 S)$ & $122.8(34.8$ in 1989$)$ \\
\hline 4.73 & $\Upsilon(1 \mathrm{~S})$ & 14.8 \\
\hline $5.25-5.29$ & Continuum and $\Upsilon(4 S)$ & 165.0 \\
\hline $5.30-5.35$ & Above $\Upsilon(4 \mathrm{~S}) \mathrm{B} \overline{\mathrm{B}} *$ scan & 63.4 \\
\hline All, 1989-1990 & & 365 \\
\hline \multicolumn{3}{|c|}{ CLEO-II data accumulated, Jan 1, 1991 - Mar. 1, 1992} \\
\hline $5.25-5.29$ & Continuum and $\Upsilon(4 S)$ & 1296.4 \\
\hline
\end{tabular}

\subsubsection{Analysis of CLEO-II dataset (B-physics)}

The outstanding performance of CESR since Fall of 1990 has provided us with a data sample for b-quark studies which is unique in both size and quality. The past year has seen the completion of several preliminary analyses of this data, including three contributed papers to last summer's Lepton-Photon conference in Geneva. The great statistical strength of our $B$ sample $\left(0.5 \mathrm{fb}^{-1}\right.$ at the $\Upsilon(4 \mathrm{~S})$ through summer of 1991 , and a roughly equal sample since, for a total of over 2 million B mesons) mandates a comprehensive understanding of the systematics of our experiment. Achieving this understanding continues to be a primary focus of our activities, with intensive studies of tracking and shower analyses, as well as significant refinement in our capabilities for Monte Carlo simulation.

In this section we discuss the present status of our b-physics analysis effort, with results based on data collected between late 1990 and summer 1991. Plans for the coming year are discussed in Section 4.

The most striking development in $\mathrm{b}$ physics in the past year does not involve $\mathrm{b}$ decay, but rather the previously reported non- $\mathrm{B}^{0} \overline{\mathrm{B}^{0}}$ decays of the $\Upsilon(4 \mathrm{~S})$. In late 1989 CLEO and ARGUS observed production of high momentum $\psi$ 's in data collected at the $\Upsilon(4 \mathrm{~S})$ resonance. The natural interpretation, continuum production of $\psi$, seemed to be excluded by both experiments' off-resonance samples. Lacking a continuum interpretation, the signal was taken to indicate an appreciable rate for direct, non- $B \bar{B}$ decays of $\Upsilon(4 S)$. This work had been done by Purdue student W-M.Yao.

The confirmation and further study of these decays was a primary goal for CLEOII analysis. Last summer we reported preliminary results on $\psi$ production in the $\Upsilon(4 \mathrm{~S})$ region. The signal for inclusive $\psi$ production in B decay (Fig. 4) was observed to be consistent with CLEO's 1987 result. The results for $\psi$ 's with momenta above the kinematic limit for production in B decay were surprising, however. Fig. 5 shows the invariant-mass distributions for dileptons with $\mathrm{x}=\mathrm{p} / \mathrm{E}_{\text {beam }}$ greater than 0.38 , for both on- and off-resonance data. Table 2 gives the yields of dileptons within $\pm 60 \mathrm{MeV}$ of the $\psi$ mass, together with sideband estimates of the background. The sum of the 1987 and 1990 off-resonance yields 


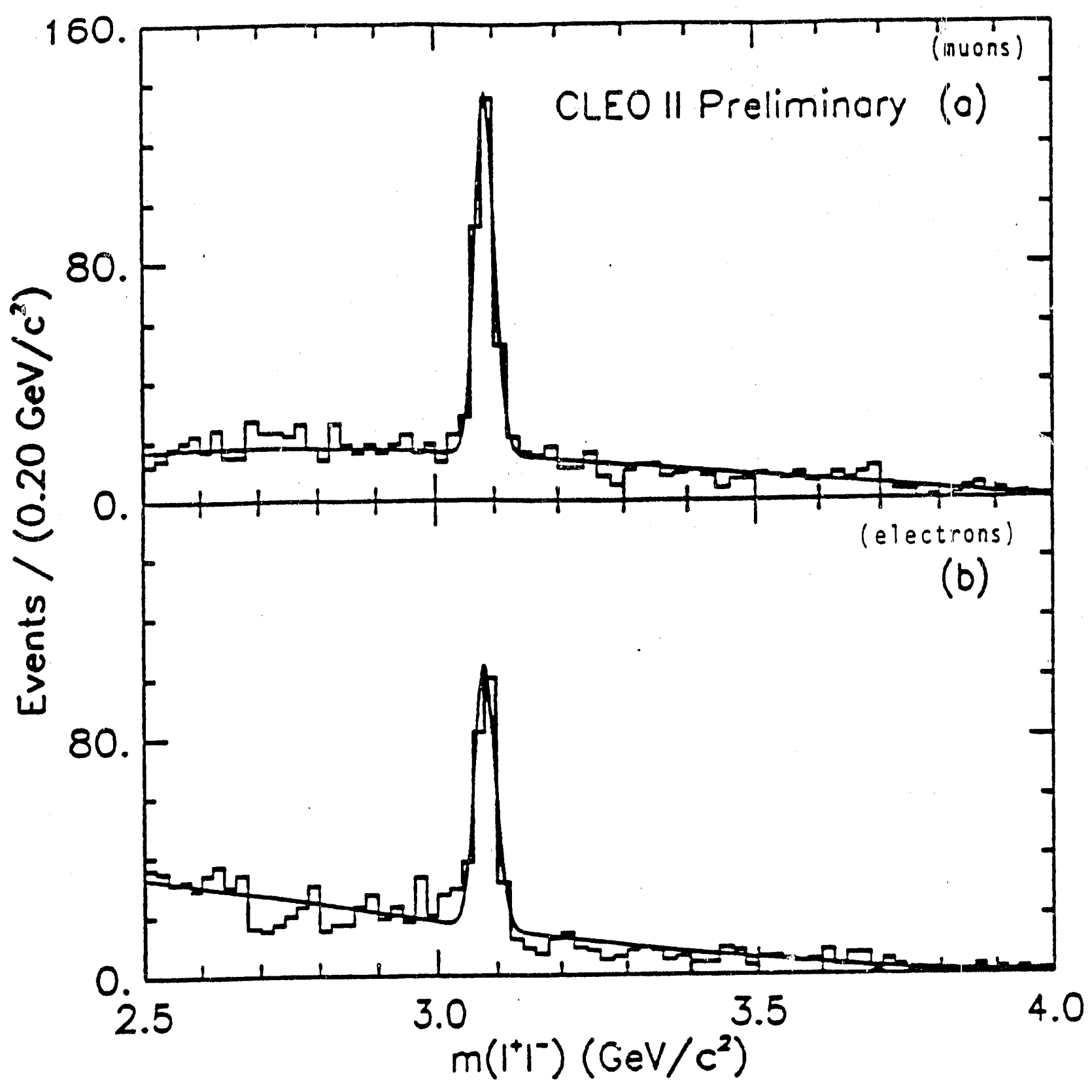

Figure 4 Inclusive $\mathrm{B} \rightarrow \psi+\mathrm{X}$ signal 


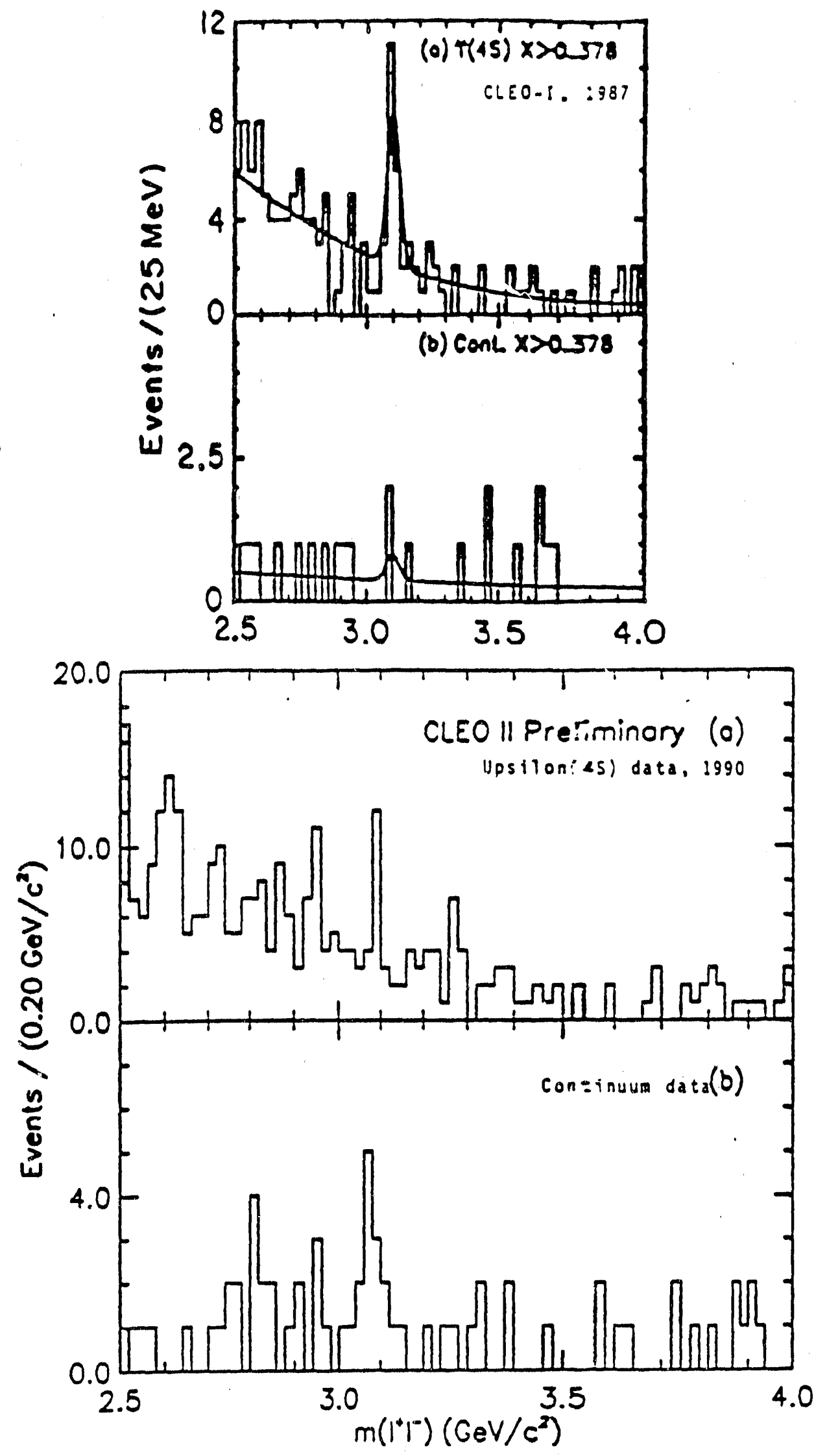

Figure 5 High momentum $\psi$ 's from on- and off- $\Upsilon(4 S)$ data samples. 
is 12 events, with a background estimate of $3.6 \pm 0.6$ events, strongly suggesting continuum production of $\psi$ 's.

TABLE 2. Summary of $\psi$ production on continuum and $\Upsilon(4 \mathrm{~S})$

\begin{tabular}{|ccc|}
\hline Data Sample & Signal & Est. Background \\
CLEO II On & 19 & $13.6 \pm 1.2$ \\
CLEO II Off & 10 & $2.9 \pm 0.5$ \\
CLEO On & 7 & $4.3 \pm 0.6$ \\
CLEO Off & 2 & $0.7 \pm 0.2$ \\
\hline
\end{tabular}

We can similarly use the on-resonance totals to reassess the evidence for direct production of high-mom sntum $\psi$ 's in $\Upsilon(4 \mathrm{~S})$ decays. At the $\Upsilon(4 \mathrm{~S})$ we observe 36 apparent high-momentum $\psi^{\prime}$, with a background of $17.9 \pm 1.3$, giving a net excess of $18.1 \pm 6.1$. Subtracting the continuum contribution, as estimated with our off-resonance yield, we find a net excess of $\psi$ mesons with $x>0.38$ of $0.5 \pm 9.6$.

Using the signals for $\psi$ 's from $B$ decays to determine the relative normalization between the two data sets ( 450 for 1990,150 for 1987, giving a factor of 3.0), we find that the 1990 off-resonance yield of high-momentum $\psi$ 's is consistent with 1987, while the 1990 on- $\Upsilon(4 \mathrm{~S})$ total falls well short. At this point we have no explanation for this other than a rather dramatic statistical fluctuation in the previous on-resonance data. The conclusion from the combined data sample is that there is no longer any reason to invoke direct non- $B \bar{B}$ decays of the $\Upsilon(4 S)$ to explain high-momentum $\psi$ production.

Our growing sample of $\psi$ 's has allowed us to conduct several studies of the details of charmonium production in $B$ decays. We have completed preliminary measurements of the branching fractions for several exclusive B-meson decay modes involving $\psi$ 's, and of the polarization of $\psi^{\prime}$ 's produced in inclusive and exclusive $B \rightarrow K^{*} \psi$ decays. The latter quantity is of interest as a probe of the dynamics of $B$. lecays and as a test of the predictions of various theoretical models. It also provides engineering input for $B$ studies in future experiments, especially in hadron colliders. In the inclusive case we measure the angle between the direction of the positive lepton in the $\psi$ rest frame, and the $\psi$ direction in the laboratory frame. Because B mesons produced at the $\Upsilon(4 \mathrm{~S})$ are slow-moving, this angle is a good approximation to the helicity angle, the angle between the positive lepton and the $B$ direction in the $\psi$ frame. In the exclusive $B \rightarrow K^{*} \psi$ study, the helicity angle is measured directly. Efficiency-corrected helicity-angle distributions for inclusive $B \rightarrow K^{*} \psi$ in several $\psi$ momentum intervals are shown in Fig. 6. At the high-momentum end, where the decay modes $B \rightarrow K \psi$ and $B \rightarrow K^{*} \psi$ are dominant, the observed polarization suggests that $\psi$ 's from $B \rightarrow K^{*} \psi$ are produced in a helicity 0 state. This is consistent with our direct measurement of a smaller exclusive $B \rightarrow K^{*} \psi$ sample, and agrees with previous reports from ARGUS.

Aided by CLEO II's excellent photon detection, we have observed exclusive and inclusive decays of $\mathrm{B}$ to $\chi_{\mathrm{c} 1}$. We used the CLEO-II calorimeter to select isolated photons in events 


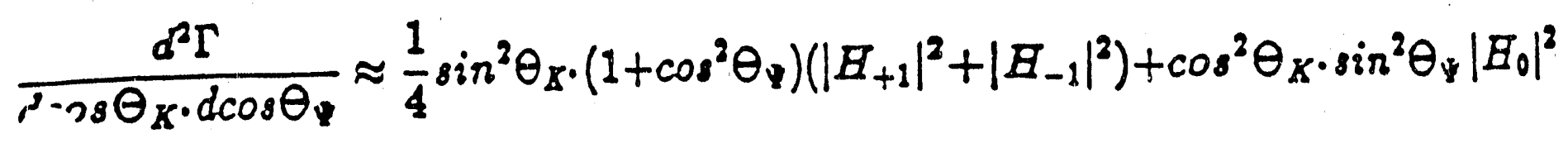
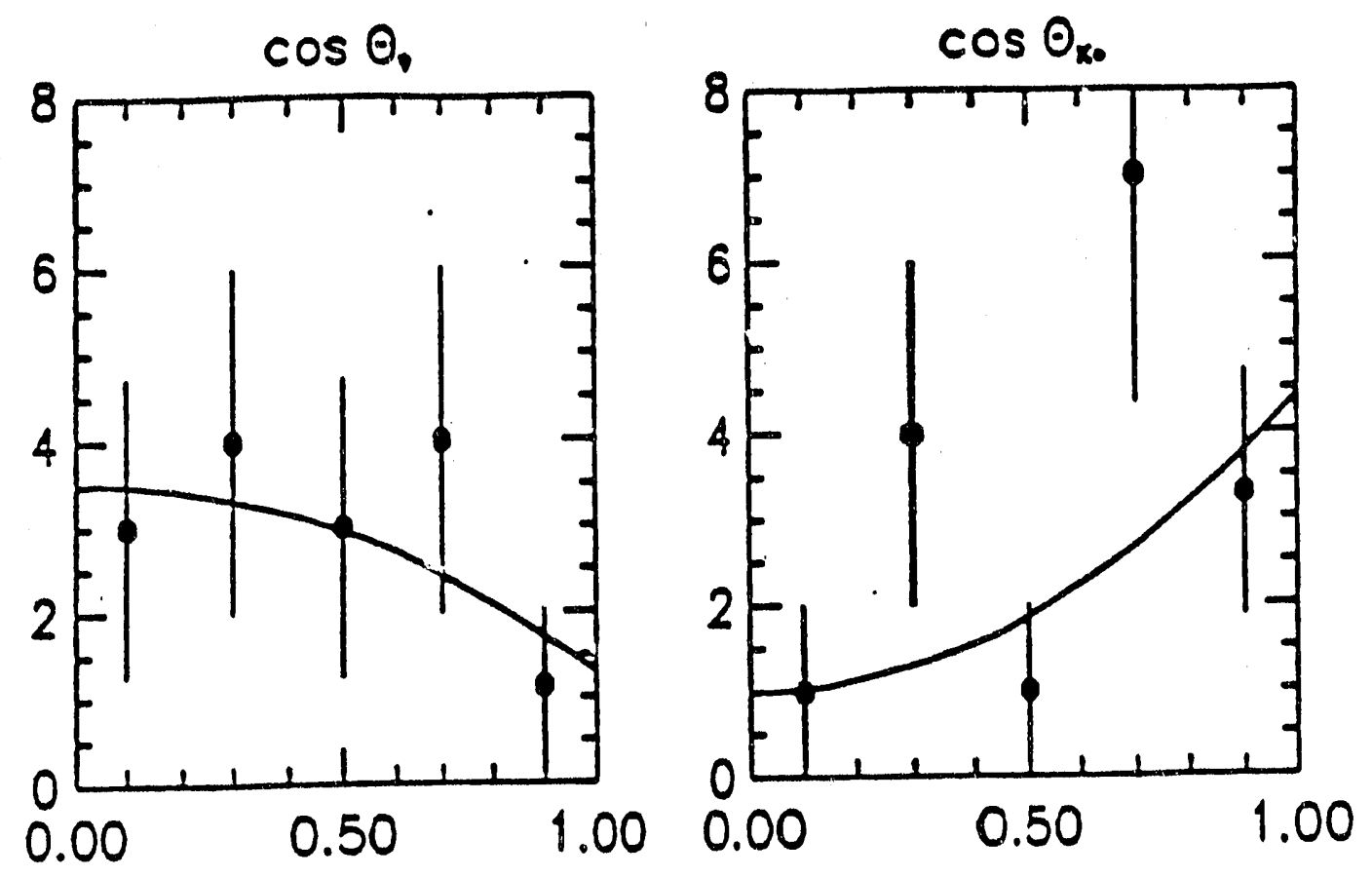

Figure 6 Helicity angle distributions for $\mathrm{B} \rightarrow \psi \mathrm{K}^{*}$. 
with $\psi$ candidates. Stringent $\pi^{0}$ sup pression was used because of the large number of $\pi^{0}$ s in both true $\mathrm{B} \rightarrow \psi \mathrm{X}$ and fake- $\psi$ events. The distribution of the mass difference $\mathrm{M}(\psi \gamma)$ $-\mathrm{M}(\psi)$ is shown for our $\Upsilon(4 \mathrm{~S})$ data in Fig. 7. An excess is evident in the vicinity of 0.4 $\mathrm{GeV}$, with the expected (from Monte Carlo) mean and width for the process $B \rightarrow \chi_{c 1} X$, $\chi_{c 1} \rightarrow \psi \gamma$. We find 42 signal entries and estimate 17.6 background within a region of 40 $\mathrm{MeV}$ centered on the nominal $\psi-\chi_{\mathrm{c} 1}$ mass difference. Using $B \rightarrow \psi X$ for normalization, we find the branching ratio for $B \rightarrow \chi_{c 1} X$ to be $(0.54 \pm 0.15 \pm 0.14) \%$. The direct decays of $\mathrm{B}$ to different charmonium states appear to be quite similar. We estimate the inclusive branching ratios for decays of $\mathrm{B}$ directly to $\psi, \psi^{\prime}$ and $\chi_{c 1}$ to be approximately $0.71 \%, 0.46 \%$ and $0.47 \%$, respectively. The momentum spectrum for $B \rightarrow \chi_{c 1} X$ is also found to be very similar to that for $B \rightarrow \psi X$. Evidence for the exclusive decay $\mathrm{B}^{-} \rightarrow \chi_{\mathrm{c} 1} \mathrm{~K}^{-}$has also been observed.

Reconfirmation of previous measurements of $\mathrm{B} \overline{\mathrm{B}}$ mixing was an important goal of early CLEO-II $\Upsilon(4 \mathrm{~S})$ running. We have now completed a preliminary analysis of inclusive dilepton production from B-meson decay. In this measurement we benefit from CLEO II's greater efficiency and lower fake background for electron identification, as well as the more than factor of 2 increase in luminosity of the 1990-91 run compared to the 1987 CLEO run. With a minimum lepton-momentum requirement of $1.5 \mathrm{GeV} / \mathrm{c}$, we find 1628 unlike-charge and 223 like-charge dileptons. After correcting for contributions from fake leptons, leptons from $\psi$ 's, continuum dileptons, and (most importantly) semileptonic decays of charm, we determine a preliminary mixing parameter value of $r=0.201 \pm 0.028 \pm 0.025$. This is consistent with previous ARGUS and CLEO measurements. Independent measurements of $B \bar{B}$ mixing, using kaon-lepton correlations, and also using tagged B-meson samples, are currently in preparation.

CLEO-II was designed to have significantly enhanced capabilities for B-meson reconstruction compared to the original CLEO detector. Miller is the leader of the $\mathrm{B}$ reconstruction effort. Our B-candidate mass distributions for a variety of exclusive decay mocies to charm are shown in Fig. 8 for charged and neutral B's, respectively. Measurements of B-decay properties using the growing sample of $\mathrm{B} \overline{\mathrm{B}}$ events tagged by complete reconstruction are in progress.

A drarnatic illustration of CLEO-II's capabilities in searches for rare B-meson decays is provided by our search for evidence of the electromagnetic penguin-mediated decay process $b \rightarrow s \gamma$. The large acceptance, excellent resolution and fine segmentation of CLEO-II's cesium iodide calorimeter have resulted in dramatic improvements in sensitivity for both inclusive and exclusive processes involving $b \rightarrow s \gamma$, compared to earlier searches.

The continuum-subtracted photon-energy distribution for the CLEO-II 1990-91 data sample is shown in Fig. 9. A signal for $b \rightarrow s \gamma$ would be visible as an excess in the photonenergy range between 2.2 and $2.7 \mathrm{GeV} / \mathrm{c}$. We observe $111 \pm 55$ photons in this interval. While this excess is suggestive, for the present we choose to quote an upper limit on the photon yield of 180 at $90 \%$ confidence level. This corresponds to an up er limit on the branching fraction for $b \rightarrow s \gamma$ of $8.4 \times 10^{-4}$. The new CLEO-II results reflect up to a factor of 14 improvement over the earlier studies.

We expect to present results on searches for inclusive and exclusive charmless semilep- 


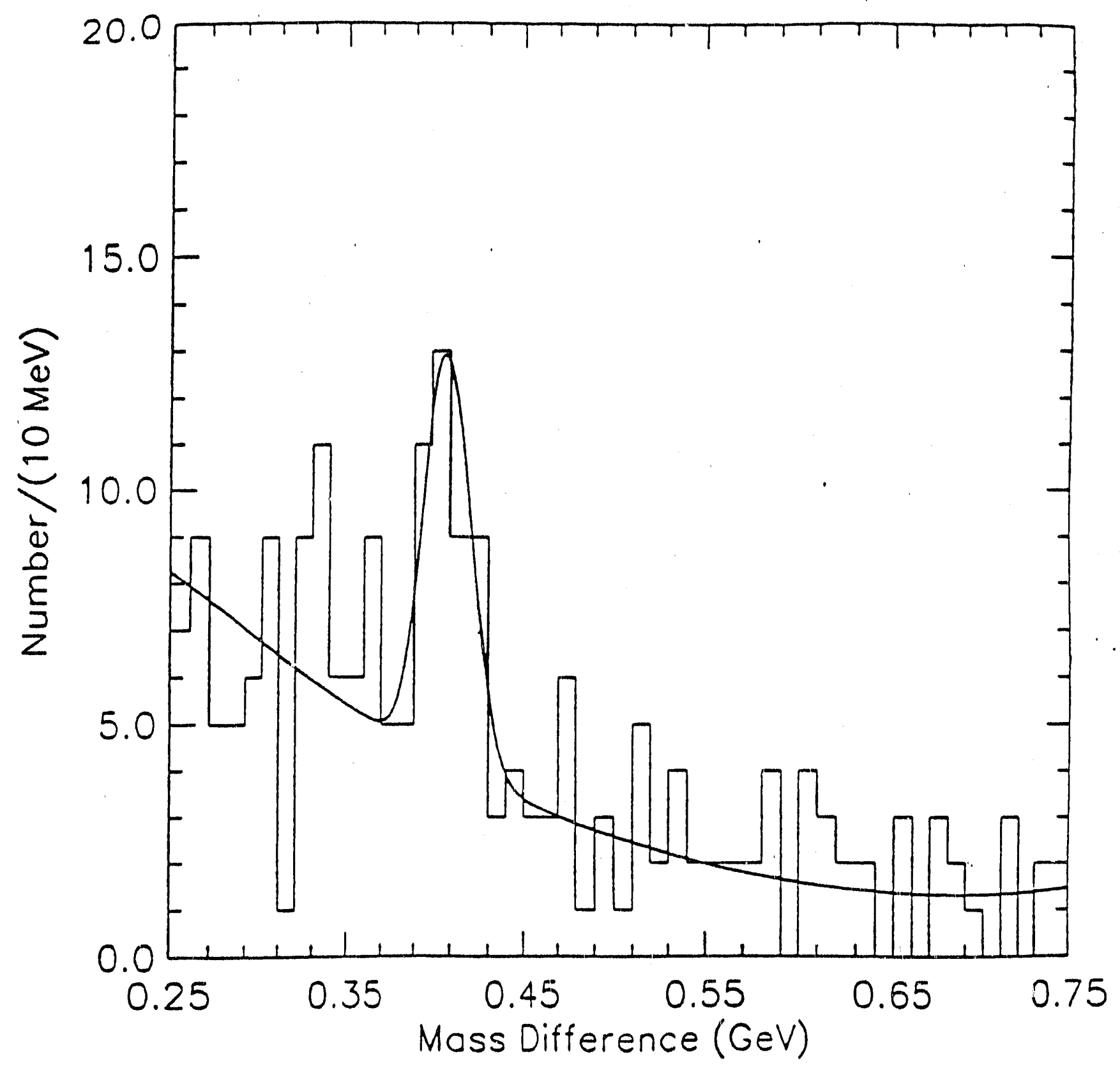

Figure $7 \mathrm{M}(\psi \gamma)-\mathrm{M}(\psi)$ mass difference for on- $\Upsilon(4 \mathrm{~S})$ data 

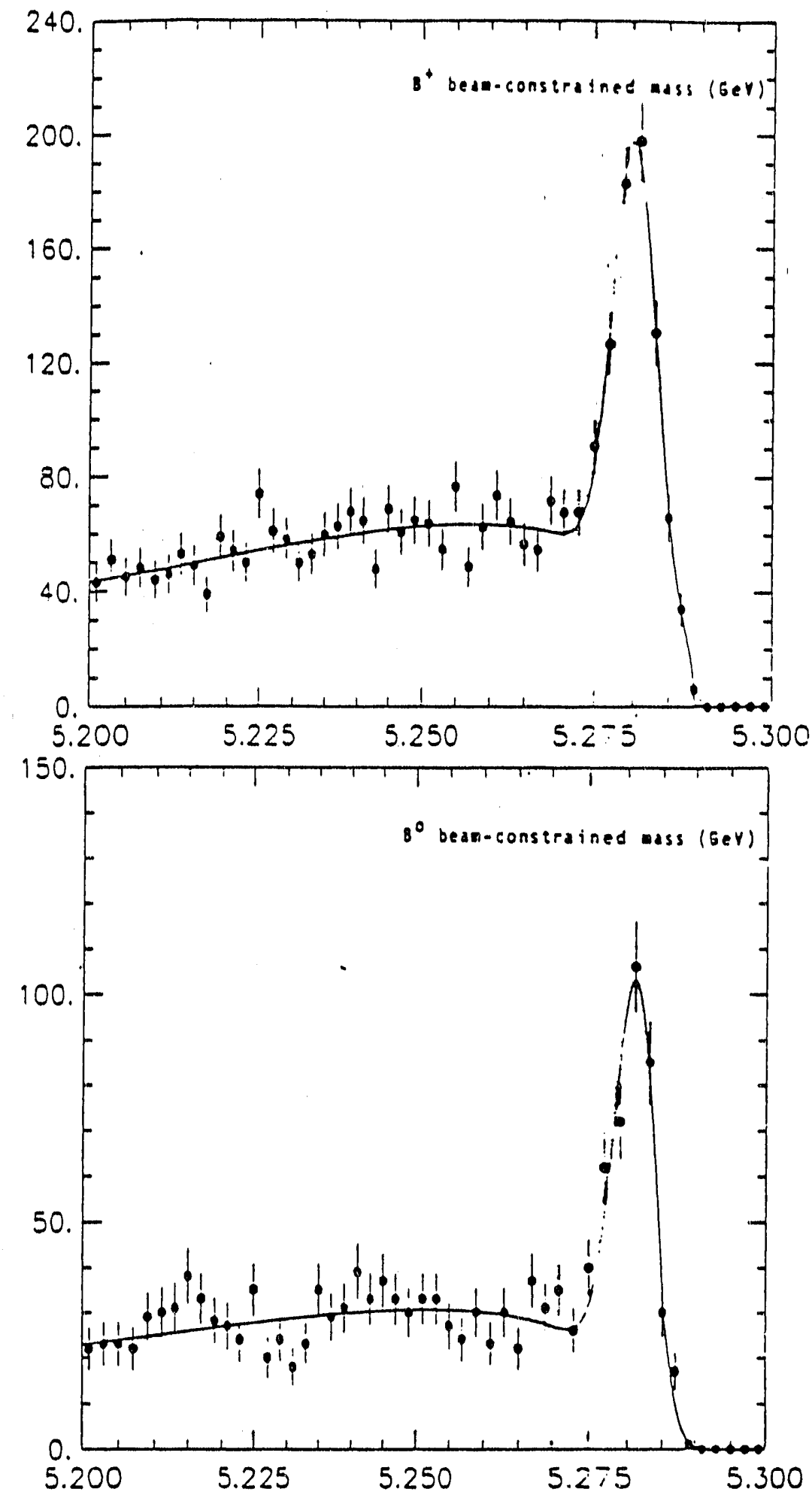

Figure 8 Beam-constrained mass for charged and neutral B-candidates 


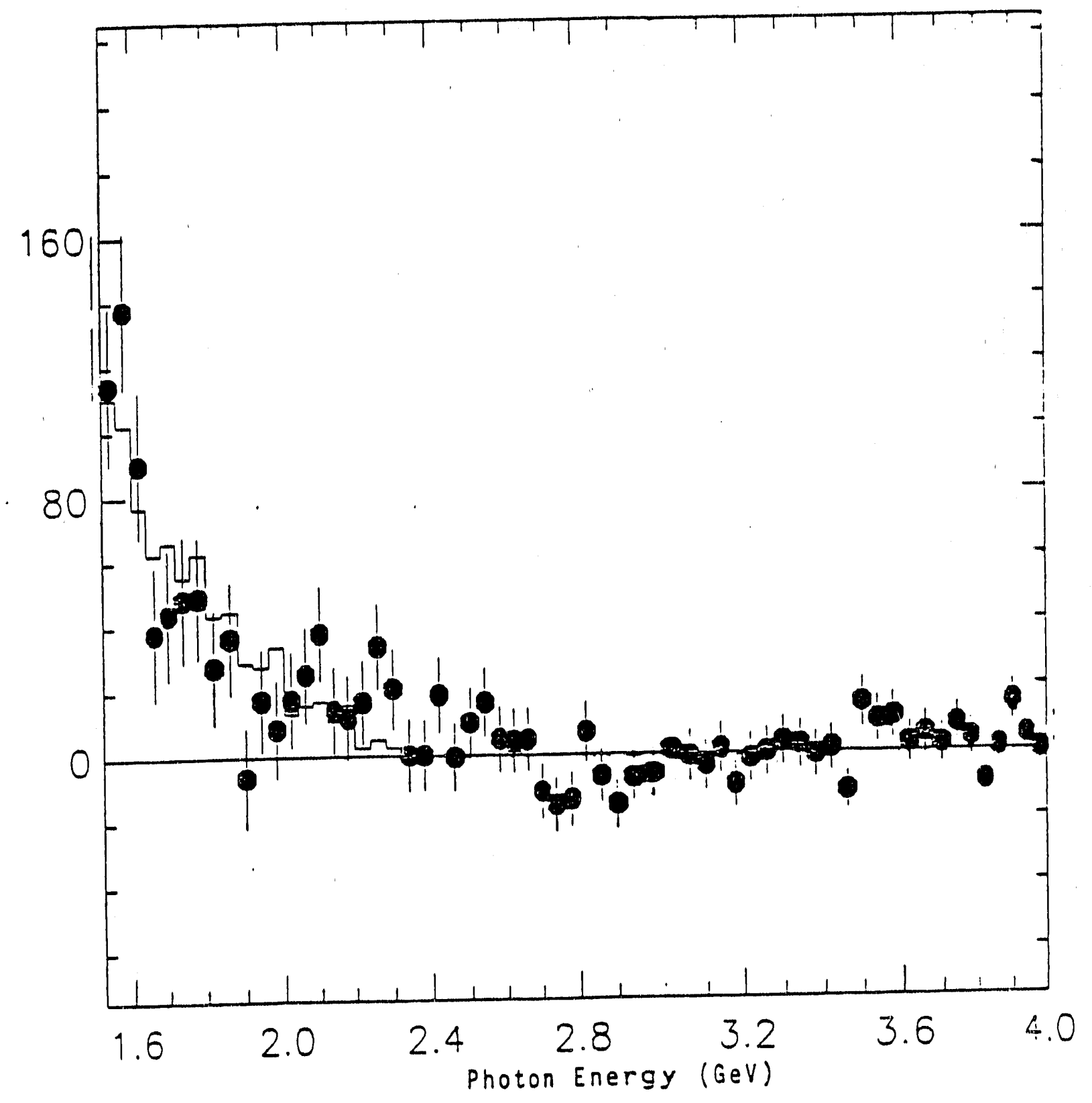

Figure $9 \Upsilon(4 S)$-continuum inclusive photon energy spectrum 
tonic $B$ decays within the next few months. We have recently completed a preliminary search for the decays $B \rightarrow \pi \pi$ and $B \rightarrow K \pi$, processes which can occur through either $b \rightarrow u$ or gluonic "penguin" couplings. The decay $B \rightarrow \pi \pi$ is of particular interest because the final state represents a potential highly detectable $C P$ eigenstates. With an expected branching ratio of $2.0 \times 10^{-5}$ (assuming $\left|V_{u b} / V_{o b}\right|$ to be approximately 0.1 ), however, the detection of this decay remains a formidable experimental challenge.

An inclusive search for two-body B-meson decays which does not distinguish between $\pi \pi$ and $K \pi$ final states is underway, with the intent of presenting a summer paper based on all our data accumulated up to that point. When particle identification is used to differentiate among the exclusive final states, we find preliminary $90 \%$ confidence-level upper limits (based on approximately half of all the $\Upsilon(4 \mathrm{~S})$ data we have written to tape) on the branching fractions for the decays $B \rightarrow \pi \pi$ and $B \rightarrow K \pi$ of $2.6 \times 10^{-5}$ and $5.7 \times 10^{-5}$, respectively.

\subsubsection{Analysis of CLEO-II dataset (resonance physics)}

Purdue student Jay Dominick is measuring branching ratios for $\Upsilon(3 S) \rightarrow \pi \pi \Upsilon(1 S), \Upsilon(3 S) \rightarrow$ $\pi \pi \Upsilon(2 S)$, and the cascade decay $\Upsilon(3 S) \rightarrow \Upsilon(2 S)+X, \Upsilon(2 S) \rightarrow \pi^{+} \pi^{-} \Upsilon(1 S)$ using the exclusive mode where the $e^{+} e^{-}$or $\mu^{+} \mu^{-}$decay of the final $\Upsilon$ is observed, as well as inclusive $\pi^{+} \pi^{-}$transitions where the final state $\Upsilon$ is not reconstructed. In both cases the properties of the $\pi \pi$ system are of considerable interest due to the previous observation of siructure different to that expected from theory.

\subsubsection{Analysis of CLEO-II dataset (charm physics)}

The charm physics program at CLEO-II has evolved along three parallel streams, concentrating on: hadronic decays of charmed mesons, hadronic decays of charmed baryons, and semileptonic decays of charmed particles. In all three cases, our high experimental sensitivity relies on our combination of high-precision tracking and calorimetry.

a. Hadronic decays of charmed baryons. . Conspicuous in the study of $\Lambda_{c}$ and $\Xi_{c}$ 's is the fact that their lifetimes are shorter than the $\mathrm{D}^{0}$ and the $D_{s}$. Therefore, here we have placed some emphasis on understanding the extent to which the lifetimes of charmed baryons may be shortened by possible exchange effects. These are believed to not be subject to the same helicity and color effects which may suppress their contributions to charmed meson decay due to the possibility of gluon exchange between the spectator and the interacting quarks. This is consistent with our observation of a relatively large rate for $\Xi_{c}^{0} \rightarrow \Omega^{-} \mathrm{K}$, which will be published in Physics Letters $B$.

Among many new modes we have discovered the $\Lambda_{c}^{+}$decay channel $\Lambda_{c}^{+} \rightarrow \Sigma^{+} \mathrm{K}^{+} \mathrm{K}^{-}$, (Figure 10), which is inaccessible through a simple spectator graph.

Much of the work in this sector has gone into optimizing our reconstruction efficiency for the decays $\Sigma^{+} \rightarrow p \pi^{0}$, and $\Xi_{0} \rightarrow \Lambda \pi^{0}$ : Many of the decay modes one would like to study (e.g., $\Xi_{c}^{0} \rightarrow \Sigma^{+} \mathrm{K}^{-}$and $\Xi_{c}^{+} \rightarrow \Xi^{0} \pi^{+}$) involve reconstruction of such lower mass, long-lived, strange baryons. Techniques to take advantage of the lifetime of such particles and how best to combine that information with the neutral particle in the decay chain are thus presently being optimized.

b. Charmed baryon semileptonic decays 


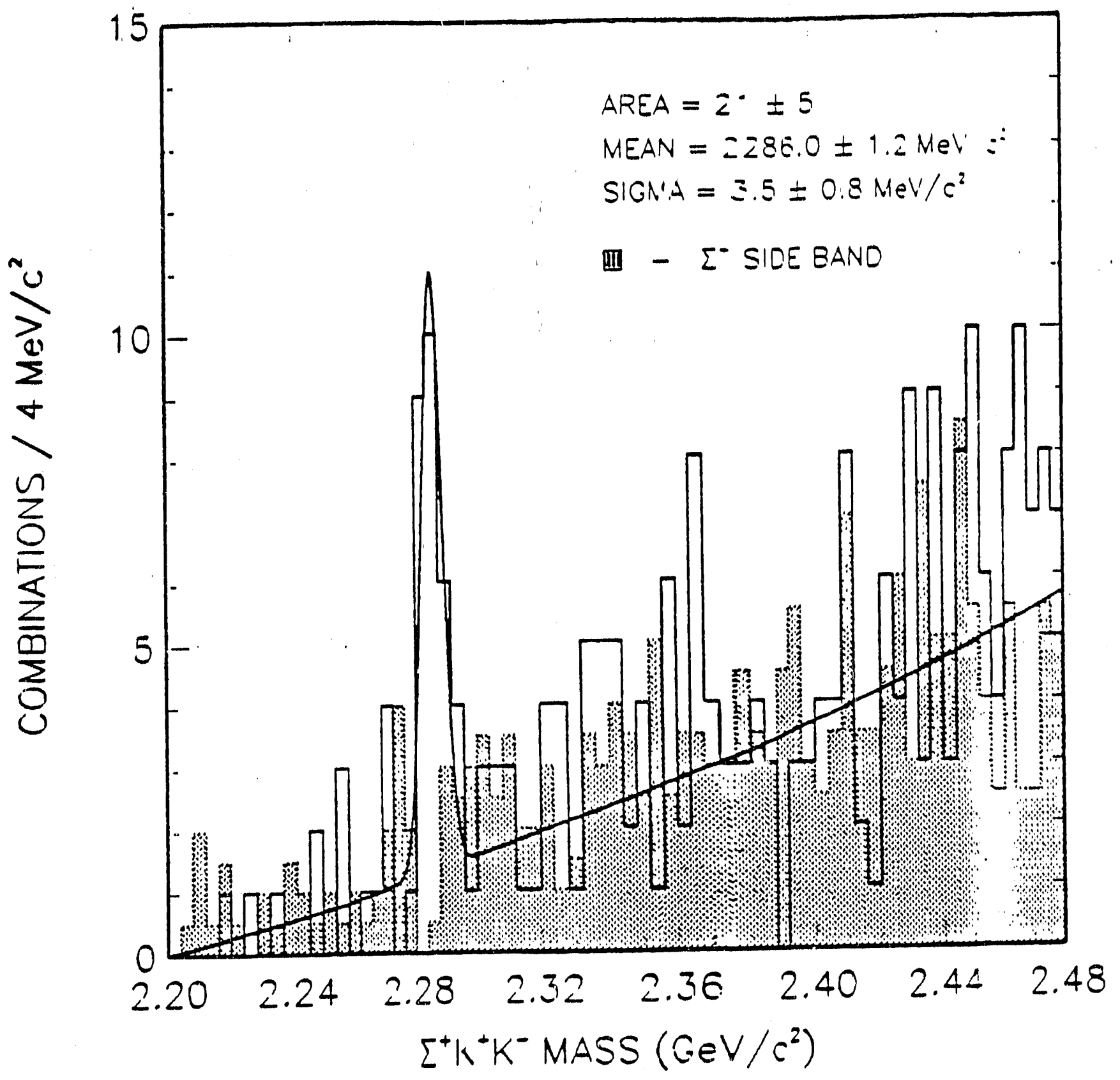

F'igure 10 Signal observed for $\Lambda_{c}^{+} \rightarrow \Sigma^{+} K^{+} K^{-}$ 
In order to understand the decay mechanisms of charmed baryons a reliable normalization is neccessary. The decay most often used to normalize is the accesible $\Lambda_{0} \rightarrow p K \pi$. However there is no reliable prediction for the rate of this decay and the experimental measurements differ by a factor two. Shipsey, in an anlys: 3 performed at Purdue in 1991, and submitted to Geneva Lepton-Photon Conference, demonstrated that a reliable normalization could be obtained by studying the decay $\Lambda_{0} \rightarrow \Lambda \ell^{+} \nu_{\ell}$ becasue the semileptonic width of the charm is already known. Using a very clean signal as shown in Fig. 11 and the known lifetime of the $\Lambda_{c}$, the first reliable normalization of the $\Lambda_{c}$ decay modes has been obtained.

\section{c. Charm semileptonic decays}

The presently tabulated sum of exclusive D semileptonic modes has been noted to fall short of the inclusive semileptonic rate, inviting the possibility of a "ore-prong deficit" in the charm sector. For the $\mathrm{D}^{0}$, the numbers for the total exclusive rate and the inclusive rate are $6.0 \pm 0.7 \pm 0.8$ and $7.5 \pm 1.1 \pm 0.4 \%$, respectively. It therefore becomes crucial to test the hypothesis that the Cabibbo-suppressed modes may be larger than expected by $\left|V_{o d}\right| /\left|V_{c s}\right|$. This could be particularly important for the decay $\mathrm{D}^{+} \rightarrow \pi^{0} \ell^{+} \nu$. Although it is Cabibbosuppressed, there are kinematic factors which may enhance the $\pi^{0} \ell^{+} \nu_{\ell}$ width. In this case, the monopole ansatz for the form-factor as a function of $q^{2}$ gives an increased $q^{2}$ regime accessible, favoring the $\pi^{0}$ rate relative to the $\mathrm{K}_{S}^{0}$ rate.

However, reconstruction of this decay is extremely difficult, owing, in part, to the plethora of $\pi^{0}$ 's produced in c $\overline{\mathbf{c}}$ decays which are unassociated with charm itself. Extracting this signal requires: a) extremely good signal-to-noise for $\left.\pi^{0} \rightarrow \gamma \gamma, b\right)$ good lepton identification over the widest momentum range possible in order to take full advantage of the soft lepton momentum spectrum from the $\mathrm{c} \rightarrow \mathrm{s}$ transition, and $\mathrm{c}$ ) extremely good photon reconstruction efficiency at low momentum in order to take advantage of the leverage afforded by requiring the $\mathrm{D}^{+}$ to be a $\mathrm{D}^{*+}$ daughter and selecting on the appropriate mass difference. We expect a signal of approximately 10 events in $1 / \mathrm{fb}$ of data, with a comparable level of background. We are sinilarly investigating the decays $D_{s} \rightarrow \phi \ell^{+} \nu_{\ell}, D_{s} \rightarrow \eta^{0} \ell^{+} \nu_{\ell}$, and $D^{+} \rightarrow \omega \ell^{+} \nu_{\ell}$. We are presenting results on some of these decays in Washington as this is being written.

\subsubsection{Analysis of CLEO-II dataset ( $T$-physics)}

The tau physics program at CLEO-II is attacking the infamous "one-prong" problem in tau decays by performing precision measurements of many of the important exclusive branching ratios. We are also attempting to measure the masses of the tau lepton and its associated neutrino.

The data accumulated by CLEO-II as of last summer amounts to $742 \mathrm{~K}$ produced taupairs. This yields a large sample of events in which both taus decay to, e.g., $\pi \pi^{0}$ (via the $\rho)$. The number of events seen is proportional to the branching ratio squared, so errors on the event counting (both statistical and systematic) are halved when the branching ratio is calculated. We are using this technique to measure $\operatorname{BR}\left(\tau \rightarrow \pi \pi^{0} \nu\right)$ and $\operatorname{BR}(\tau \rightarrow e \nu \nu)$. We normalize to luminosity (known, using large angle Bhabhas and $\gamma \gamma$ events, to 1.5\%) times cross-section (predicted with a theoretical error of $1-2 \%$ ). We believe that we can understand our event triggering and reconstruction efficiency with an absolute systematic error of better than $4 \%$ (through detailed comparison of the data to Monte Carlo predictions), giving systematic errors on these two branching ratios of $2 \%$. 


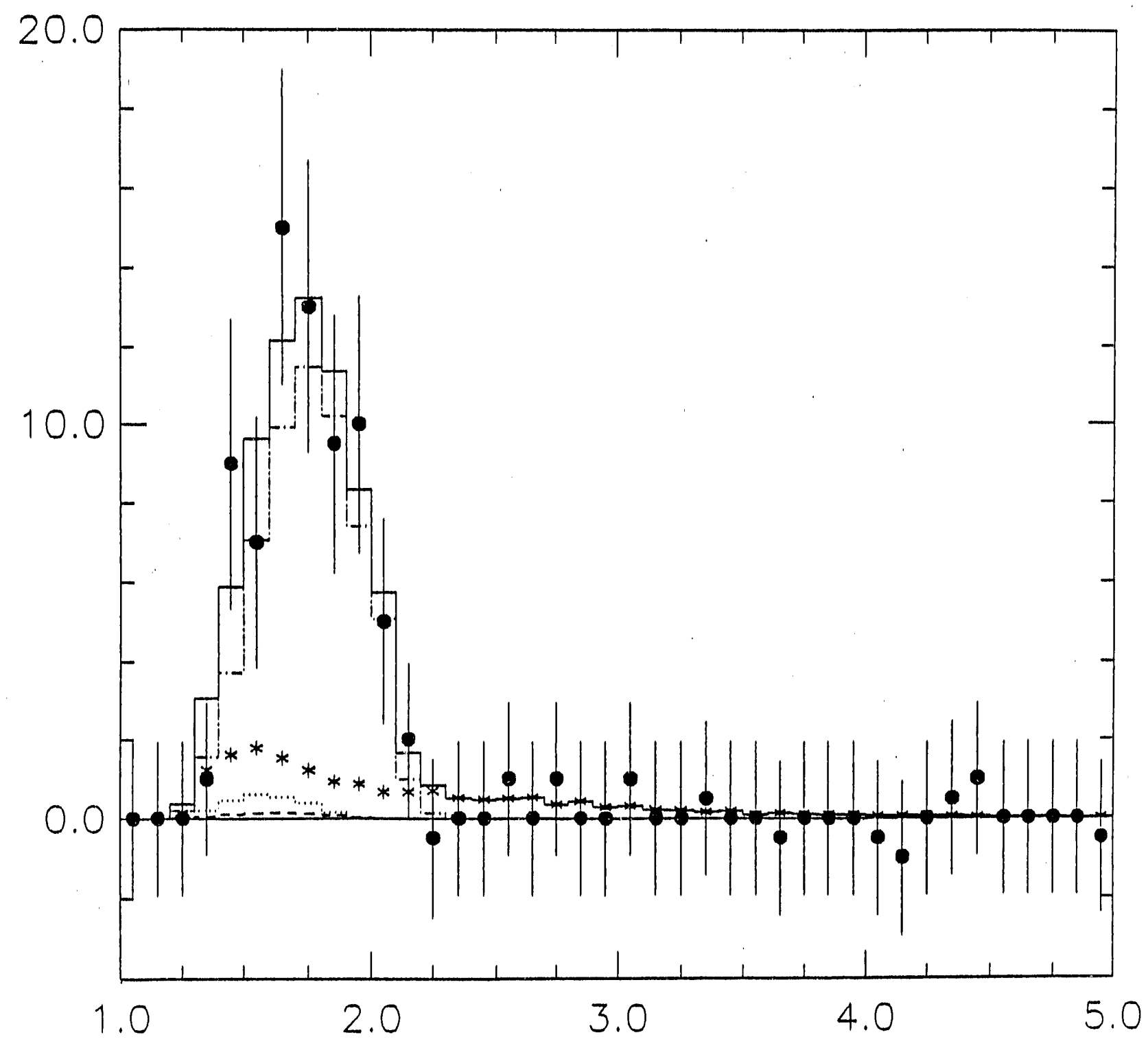

Figure 11 Invariant mass for $\Lambda l$ pairs 
The two $\pi^{0}$ 's in an event of the type $\rho^{+} \nu-v s-\rho^{-} \nu$ stand out clearly above background, as can be seen in Fig. 12. We exploit the power of our calorimeter by using it to not only reconstruct $\pi^{0}$ and $\eta$ mesons efficie.stly, but also to veto events containing extra unused showers (mainly from feed-down from higher-multiplicity tau decays and $\mathrm{q} \overline{\mathrm{q}}$ ). In this case, no extra unused showers with energy greater than $80 \mathrm{MeV}$ may appear in the calorimeter.

The branching ratios to hadronic modes containing more than one $\pi^{0}$ are determined by me asuring their ratios relative to the one- $\pi^{0}$ mode, so that systematic errors associated with the tracking and the trigger efficiency mostly cancel. Here, we tag the tau-pair event by requiring one side to go to $e, \mu$, or three-prongs, and reconstruct one or more $\pi^{0}$ 's on the other (one-prong) side. Modes with as many as four $\pi^{0}$ 's are clearly seen, recoiling against a leptonic tag. We plot in Fig. 13 the invariant mass of the hadronic system in such events. The background from fake- $\pi^{0}$ 's can be estimated from the sidebands and subtracted. There is virtually no contamination from $\mathrm{q} \overline{\mathrm{q}}$ events; remaining background comes from migration from other tau-pair modes (such as $\pi^{+} \pi^{0} \nu-v s-\pi^{-} 3 \pi^{0} \nu$, where the $\pi^{+}$fakes a muon). The migration matrix, estimated from the Monte Carlo, has very small off-diagonal components.

It has been suggested that a possible source of missing one-prong decays is modes containing $\eta$ mesons. Theoretical expectations for these branching ratios, however, are small. We have recently established the first clear observation of $\tau \rightarrow \eta X$, in the decay $\tau^{+} \rightarrow \pi^{+} \pi^{0} \eta \nu$. We reconstruct the $\eta$ meson in all three of its main decay modes $\left(\gamma \gamma, \pi^{0} \pi^{0} \pi^{0}\right.$, and $\left.\pi^{+} \pi^{-} \pi^{0}\right)$, and a variety of different tags, and obtain consistent branching ratios in all cases. The signal from the decays $\pi^{0} \rightarrow \gamma \gamma, \eta \rightarrow \gamma \gamma$ can be seen in Fig. 14. We obtain a branching ratio on the same order as expected from theory. We also have limits on many other decay modes containing $\eta$ mesons. It is clear that modes containing $\eta$ mesons do not contribute sinificantly to the one-prong branching ratio.

The mass of the tau lepton will be measured to good precision by the BES storage ring and detector at Beijing. In case the result is significantly different than the old SPEAR result (in the PDG), it will need independent confirmation. We are working on a novel approach to measuring the mass by looking at events in which both taus decay to fully reconstructed hadronic systems. In that case, the maximum kinematically-allowed mass of the tau can be calculated on an event-by-event basis (although it can be thrown off by the presence of initial-state radiation and misidentified leptonic decays). The observed distribution from $\rho \nu-v s-\rho \nu$ events (Fig. 15) exhibits a reasonably sharp edge near the nominal tau mass. We are attempting to calibrate the position of that edge using Monte Carlo, and hope for an absolute precision on the oider of a few $\mathrm{MeV}$. 


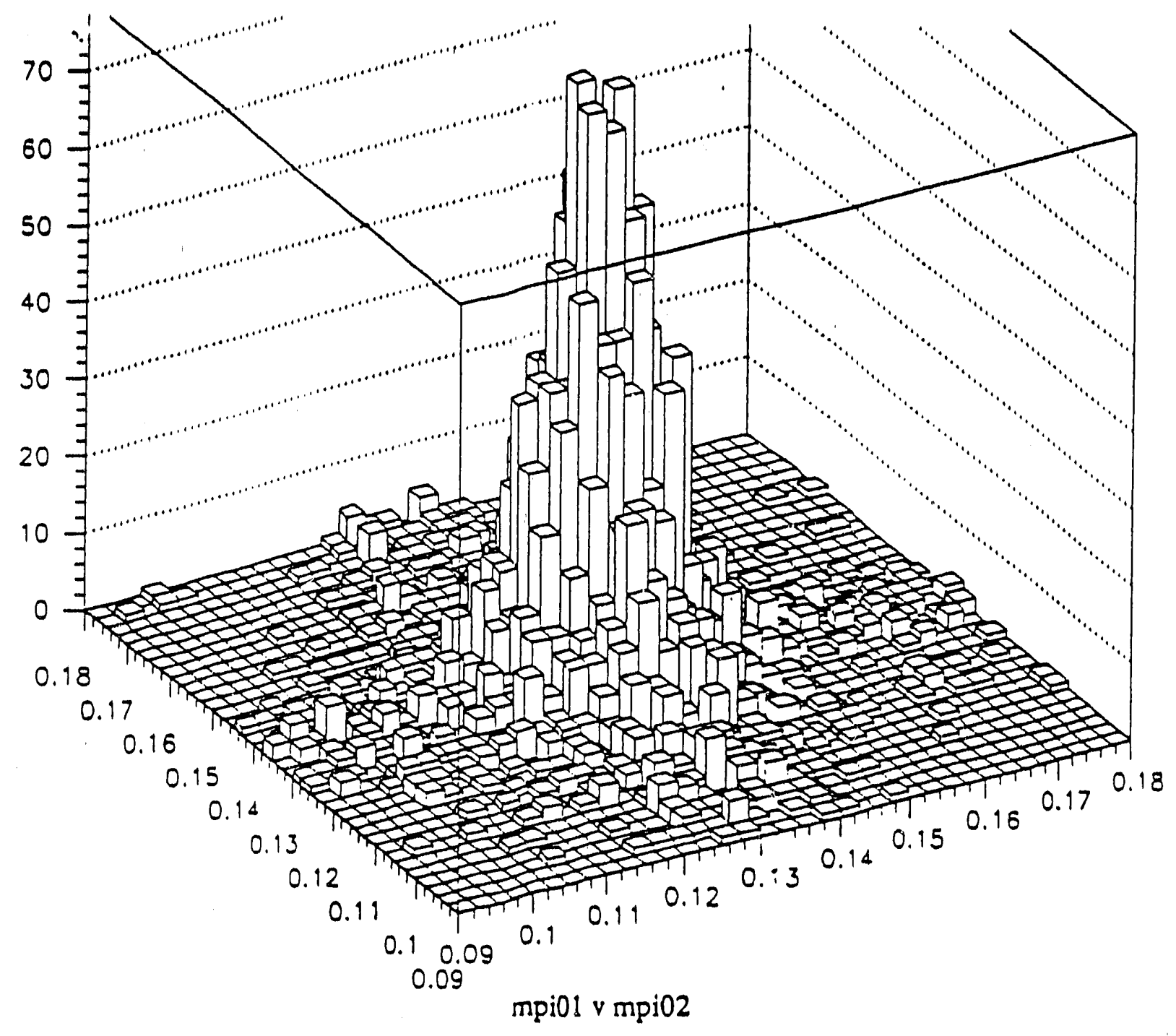

Figure $12 \mathrm{M}_{\gamma \gamma}$ vs. $\mathrm{M}_{\gamma \gamma}$ for 1-vs.-1 events, where one photon pair is closer in angle to one track and the other pair to the other track. 


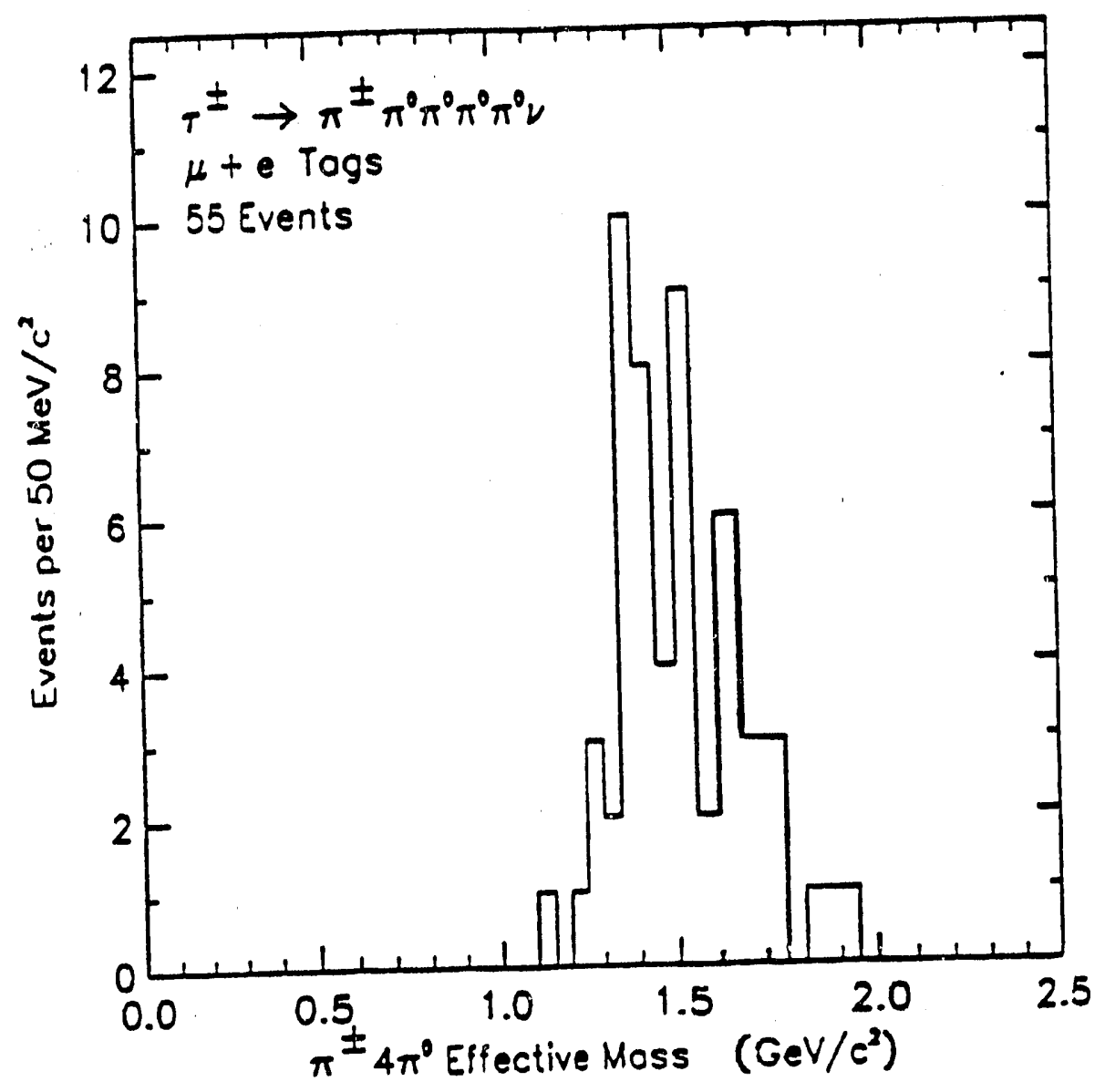

Figure $13 \mathrm{M}_{\pi 4 \pi^{0}}$ recoiling against a leptonic tag. 


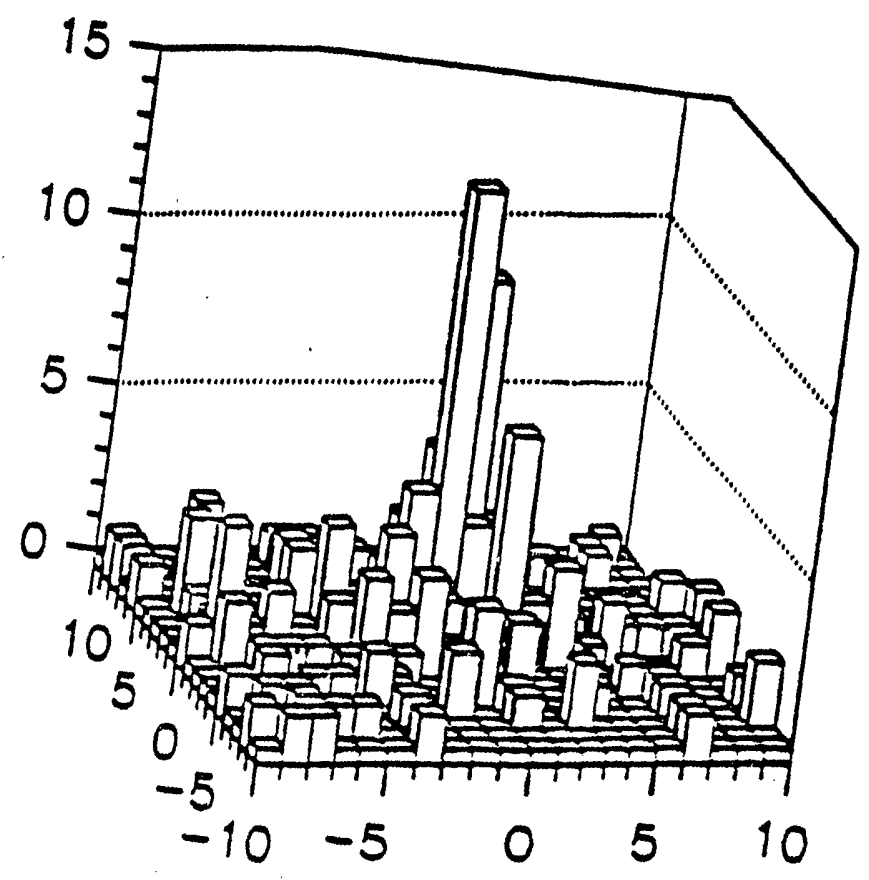




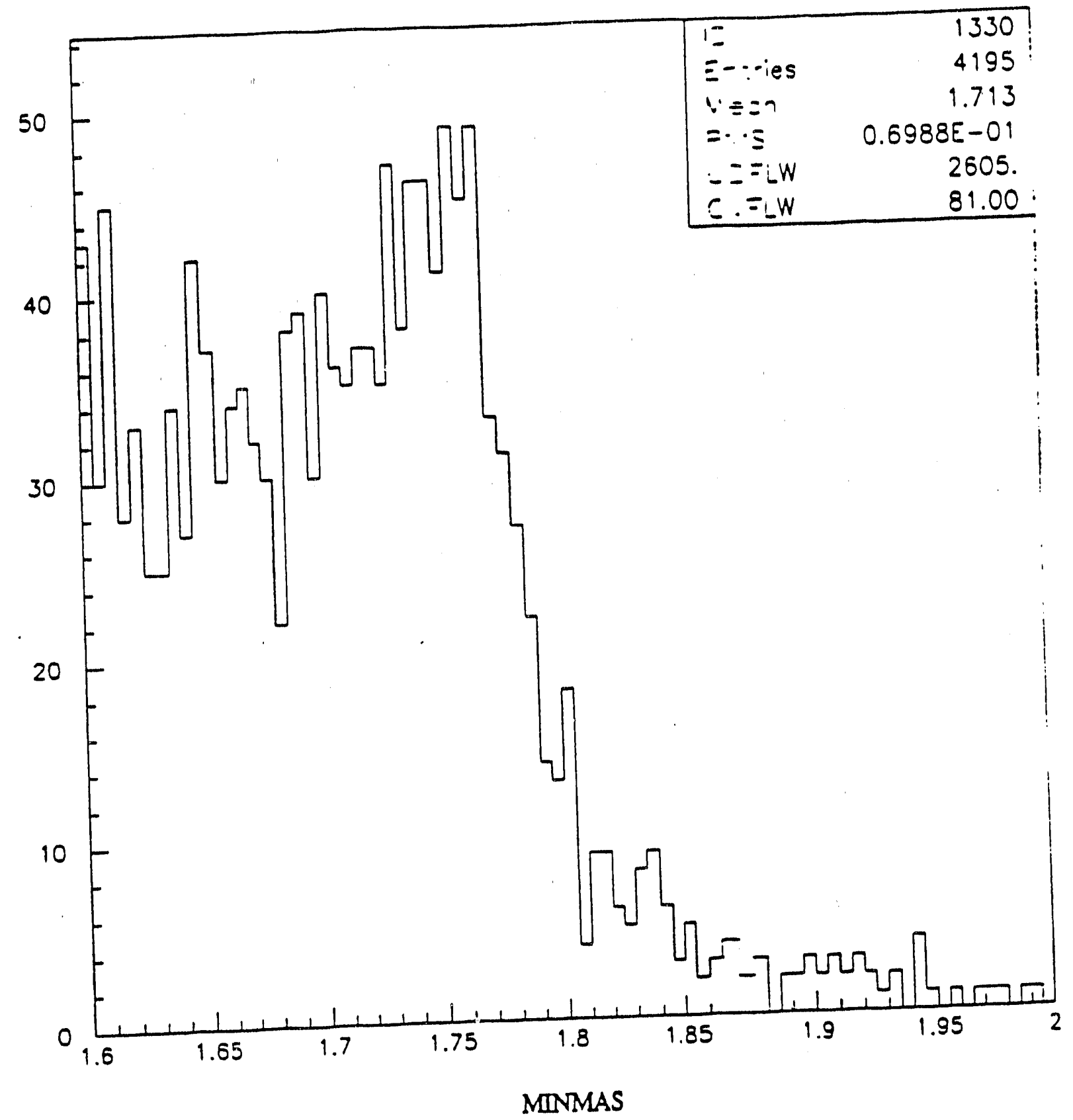

Figure 15 Maximum kinematically allowed mass of the tau lepton, calculated on event-byevent basis in $\rho \nu-v s-\rho \nu$ events. 


\section{CHAPTER 2 \\ Gas Microstrip Detectors}

The Purdue Gas Microstrip Detector (GMD) research program, initiated in 1991, is involved in research, development and evaluation of the GMD as a suitable technology for tracking at the SSC and B Factories. GMD prototype chambers have achieved $20 \mu \mathrm{m}$ spatial resolution, a detector memory comparable to the SSC and B Factory crossing time and MRad radiation hardness. It is likely that this is the only technology that will operate well at small radii and small angles as the SSC luminosity increases toward $10^{34} \mathrm{rm}^{-2} \mathrm{~s}^{-1}$. The GMD is therefore an attractive upgrade to central tracking for the $\mathrm{SDC}^{4}$ experiment as well as currently being the candidate technology for intermediate angle tracking in the SDC baseline design. The GMD also represents a solution to the low mass, high accuracy requirements of a vertex detector for a B Factory. These two seemingly disparate applications have led to a research program at Purdue with the aim to build the first prototype $\left(0.1 \mathrm{~m}^{2}\right)$ cylindrical GMD with two-dimensional readout to be installed in the CLEO experiment at CESR in 1994. This device will make a valuable contribution to CLEO tracking and trigger immediately, and is a natural part of the upgrade program towards a B Factory. Simultaneously it represents perhaps the only opportunity for a test of a GMD in a collider experiment before SSC turn-on.

The GMD group consists of Shipsey, graduate student Miao and undergraduate student Menon. As a first step, the immediate aim of the research program at Purdue is to develop a plastic GMD with novel two dimensional position information by fall 1992, accordingly we have been involved in the folowing activites in the past year:

- We have assembled our first GMD using a $1.2 \mathrm{~mm}$ glass substrate built using electronbeam lithography at RAL. This detector has been housed in a suitable shell at Purdue to provide the necessary gas, high voltage and readout connections and is currently under test. A photograph of the detector is shown in Fig. 16. A typical pulse from the detector and a pulse-height sepctrum are shown in Fig. 17. We will use this detector as a bench-mark for comparison with new detectors we are currently building.

- Award of Purdue Research Foundation Grant to Shipsey and Miao entitled "Gas Microstrip Research for SSC and B Factories".

- Search for a suitable plastic substrate. A key parameter in the design of a GMD is the resistivity of the substrate. In collaboration with Texas A\&M and Rochester SDC colleagues we have begun to measure the resistivity of a variety of suitable plastics.

- Authors (with OSU) of a GMD proposal for a B Factory which was included in the Cornell B Factory proposal submitted to NSF in February 1991.

- Presentation of a proposal to insert a GMD in CLEO II at the workshop: "Vertex Detection at $10 \mathrm{GeV}$ " at Cornell August 1991, and at the SDC Intermediate Angle Tracking Detector (ITD) Meeting at RAL, October 1991.

- Member of the SDC ITD group. Joint author of ITD Conceptual Design Report Fall, 1991, resulting in GMD being included in the baseline design of the SDC detector Feb., 1992. 


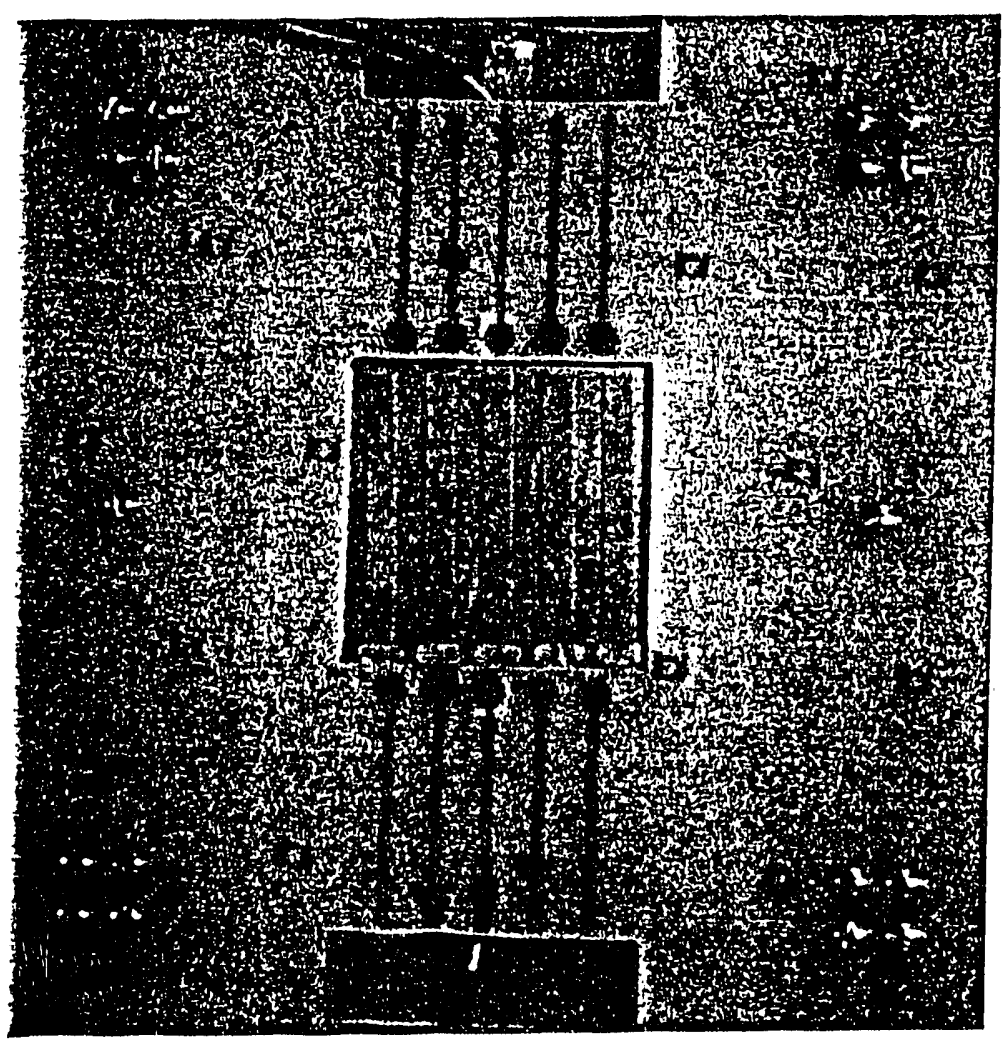

Figure 16 The Purdue GMD . 


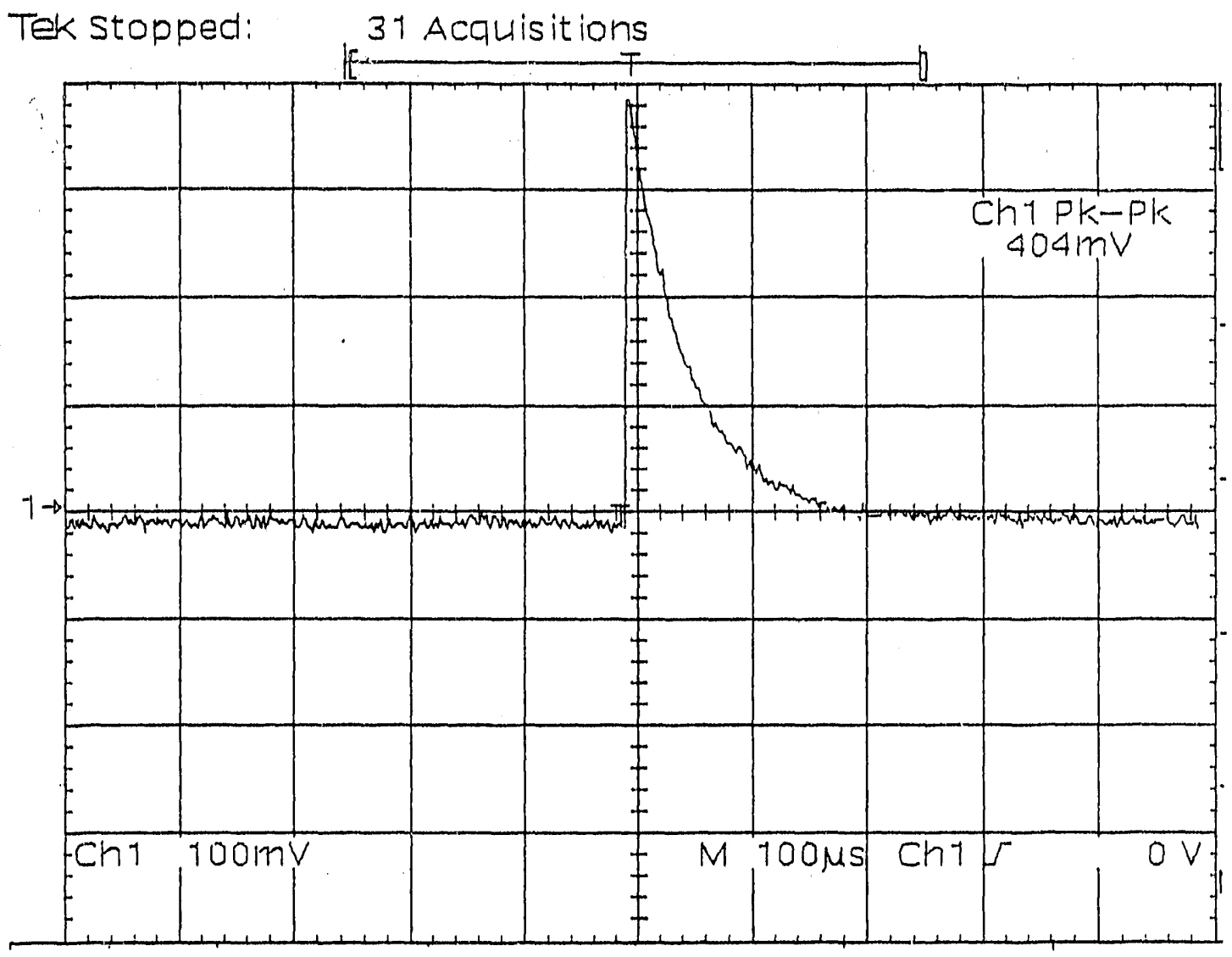

Figure 17 A pulse from the Purdue GMD. 
- We are the only group in SDC or CLEO to have instrumented the backplane of the substrate with cathodes for true two-dimensional position reconstruction.

- We have identified a company in New Hampshire that will build additional chambers using lithography and laser ablation. Laser ablation may represent the best way to produce the large area detectors required for SSC. We are the only group exploring this possibility.

- We begun a collaboration with Solid State faculty and the department of Electrical Engineering at Purdue with the aim of in-house fabrication of small scale plastic substrate prototype detectors using lithography and novel plastics such as ion-implanted kapton.

- The high rate operation of a GMD is crucial for SSC. We are preparing to test detectors in high intensity X-ray sources at Purdue.

- A final proposal for a cylindrical GMD insert into CLEO II is being prepared (with OSU) for presentation to the CLEO collaboration. It is expected that the insert will be built at Purdue in 1993 and installed into CLEO in 1994. The experience gained with this detector will provide a good foundation for the further development of a GMD for SDC and B Factories.

The timescale is short, funding for technical support staff and a post doc are critical if the opportunity this program has identified is to be grasped.

\subsection{CONCLUSIONS.}

CLEO-II enjoys the unique combination of being a high-precision detector in an unparalleled luminosity environment. Over the last year, we have considerably refined our understanding of detector performance, and begun producing physics results from the experiment. We intend that CLEO II will remain pre-eminent in $B$ physics until the advent of the SSC/B Factory era. To achieve this, we have embarked on a neccesssary program of detector $R \& D$ and upgrades.

\section{CHAPTER 3 \\ Particle Astrophysics}

The Purdue particle astrophysics group is involved in projects searching for astrophysical sources of high energy particles in the energy regime well beyond the limits of present accelerators. Although it has been established that the cosmic ray spectrum extends to energies of at least $10^{22}$ electron volts, there are currently only conjectures proposed to suggest sources and acceleration mechanisms for particles whose energies are greater than a few teravolts. Pop lar galactic candidates for sources are pulsars or black holes in compact binary systems, whereas active galactic nuclei are strongly suggested extra galactic sites of energetic radiation. There is essentially no information on the acceleration mechanism; although several models have been explored, none are particularly compelling. Of particles emitted by an energetic astrophysical object, only photons and neutrinos can be traced back to their origin and can maintain any time coherence characteristic of the source. Photons can derive 
from either accelerated charged particles or the decay of neutral hadrons, whereas energetic neutrinos are generated only in hadronic processes. In addition, the subsequent absorption by any intervening medium is distinctly different for photons and neutrinos. Hence, photon and neutrino beams convey complementary information about both source and acceleration mechanisms.

Although high energy gamma rays are a tiny component of the cosmic ray flux, that they propagate undefiected allows resolution from background photons by looking for excess counts in a small angular region around the selected source. This traditional method of discovering sources is useful in experiments where the signal to noise ratio is very large, as in satellite borne X-ray detectors, but has serious shortcomings in ground-based atmospheric Cherenkov experiments which attempt to resolve low flux sources against an intense background of electromagnetic showers initiated by relativistic hadrons. Consequently, until recently, results in very high energy gamma ray astronomy have been of marginal statistical significance and very seldom verifiable through repeated observations. The finite shower size delimits angular resolution thus the possibility of background subtraction for finding a small signal. However, the imaging technique pioneered by the Whipple collaboration has been remarkably successful in both improving resolution and producing repeatable results. The imaging method utilizes characteristic differences between gamma-induced and hadroninduced electromagnetic cascade patterns in the atmospheric air showers to reject more than $99 \%$ of the background hadron-induced showers. Observations the Crab Nebula utilizing this technique have produced results with an improvement of more than an order of magnitude in statistical significance over prior results and have been subsequently verified in following years. Furthermore, the Whipple results on the Crab have been recently confirmed by two French groups, THEMIS and ASGAT, the latter group including Palfrey as a member. One peculiar feature of these recent findings is that the signal from the Crab is seen only in a DC mode; the period of the pulsar does not modulate the data at any significant level above 100 $\mathrm{GeV}$, the minimum detection energy of the Whipple telescope. Both French groups corroborate this observation at somewhat higher energies, but more importantly, this finding has been confirmed at lower energies by detectors on the Compton Gamma Ray Observatory, where both the pulsed and DC component of the Crab are seen with the pulsed component observed to be falling more rapidly with energy. For the first time, a consistent picture of an astrophysical gamma ray signal in the few $\mathrm{GeV}$ to several $\mathrm{TeV}$ energy regime has been established. Subsequent observations in the next few years will be able to examine whether there is an episodic feature characteristic of the Crab Nebula, truly the first " $\mathrm{TeV}$ gamma ray candle".

Teravolt gamma ray astronomy is emerging as a new and viable branch of particle astrophysics. As has often been the case in high energy physics and especially astrophysics, discovering unanticipated phenomena is likely to be the most fascinating outcome of these new detectors.

Currently, the main focus of the Whipple collaboration is the implementation of stereoscopic viewing with twin imaging air Cherenkov telescopes shown in Fig. 18. This program is well underway with a second telescope having been brought on line during December-January of 1991-1992. Consisting of hexagonal mirrors with a 20 diagonal on a parabolic back plane of approximately 11 meters diameter, the new telescope is about 142 meters from the origi- 

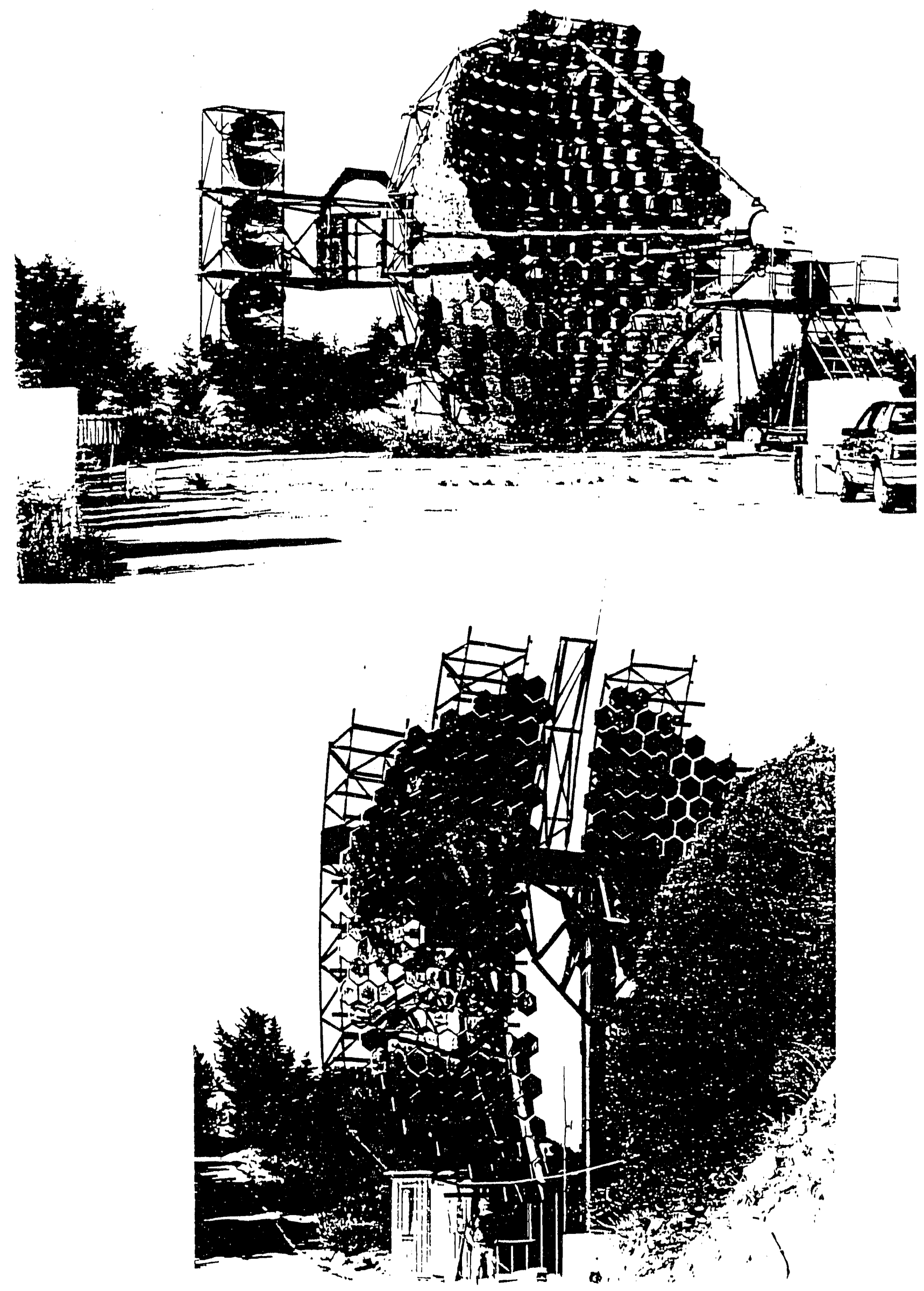

Figure 1810 and 11-meter Whipple Air Cherenkov Telescope. 
nal 10-meter Whipple telescope. Presently, coordinated observing with the two telescopes is being fine-tuned. In addition, plans for upgrading the electronics, data acquisition hardware and software, and improving the link between the detectors is underway. With the imaging technique, the pattern of Cherenkov radiation from gamma-induced showers recorded by the photomultipliers has an approximately elliptical shape for which a principal axis can be well determined; in contrast, hadron-induced showers are patchy and rarely produce elliptical patterns. With simultaneous stereoscopic viewing, the patterns recorded in each telescope are independently fitted for the same event; the two patterns with axes can be superimposed with the intersection of the axes determining the point of shower origin. The accuracy of shower location by this method on the order of 2 arc-minutes, significantly more precise than satellite borne $\mathrm{X}$-ray or $\gamma$ - ray measurements. This technique has been tested on the Crab Nebula with the 10-meter telescope in a non-simultaneous mode by taking data with the telescope pointing $0.4^{\circ}$ off-source, then locating the source to 2 arc-minutes by intersecting the fitted image axes. This accuracy is unprecedented in high energy astrophysics.

In addition to working with the air Cherenkov telescopes, we have installed the initial detectors of an air Cherenkov array as illustrated in Fig. 19. This array consists of units of four photomultipliers each which are exposed directly to the atmosphere; currently there are four units in place with and additional four being tested in our lab. This is a unique detector which will operate in coordination with the Whipple air shower array and the Whipple air Cherenkov telescopes, but at a higher energy threshold of about $50 \mathrm{TeV}$. The array will utilized programmable timing delays to effect coverage of two to three observing windows of about $5^{\circ}$, one centered on the selected source and the others serving as background monitors. This air Cherenkov array will thus track a given source with no moving parts.

We have designed and fabricated the constant-fraction discriminators and the programmable delay modules, as well as additional data control modules for this project. Most of the remaining electronics has been recycled from previous experiments. The initial test data is being used to fine-tune timing off-sets and as input to fast plane fitting algorithms.

The collaboration of Palfrey with the French group on the ASGAT experiment based in the French Pyrenees has continued and this year they have seen their most exciting result; a five sigma counting excess on the Crab Nebula. A major ingredient of this successful observation was a change in operating procedure based upon the point-spread-function calculation of Palfrey. In addition, Palfrey has continued with analysis of data taken on Her $\mathrm{X}-1$, resolving no signal as yet.

The graduate students are in the late stages of data analysis for their thesis work. Neal Berezny is pursuing a comprehensive study of all the Haleakala data taken on Her X-1. He is exploring an analysis technique to improve weak signal extraction from the sharp timing data. Galen Zirnstein has culled and recalibrated the Haleakala data on the Crab Nebula, producing a two-fold increase in usable information. He is developing a new set of event parameters which will enable usage of shower pattern characteristics in event selection. 


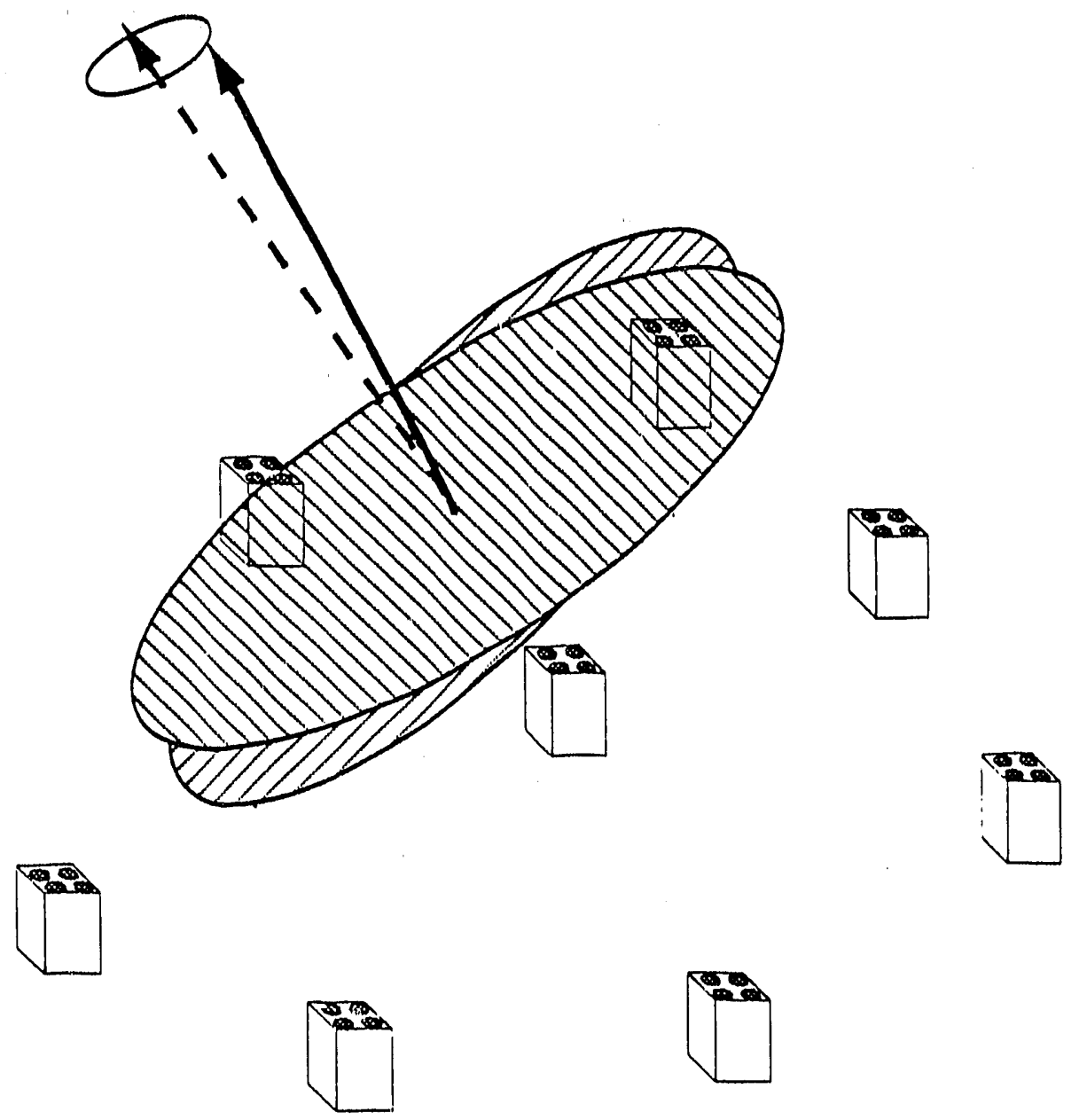

Figure 19 The Air Cherenkov Array on Mt. Hopkins. The first four units of this array have been installed and tested. Each unit contains four photo-multipliers. By incorporating programmable delay modules, a small area of the sky can be selected for tracking, thereby considerably reducing the counting rate from night sky backgrounds. This detector will operate in coordination with an extensive air shower array at the same location. The second four units have been built and are being tested in our lab. 


\section{REFERENCES}

1. Y. Kubota et al., Oornell preprint CLNS 91/1122, submitted to Nucl.Inst,and Meth. A.

2. C. Bebek et al. Nucl.Inst.and Meth, A302 (1991).

3. D. Bortoletto et al., Nucl.Inst.and Meth. A, in press.

4. The Solenoidal Detector Collaboration EoI, May (1990). 

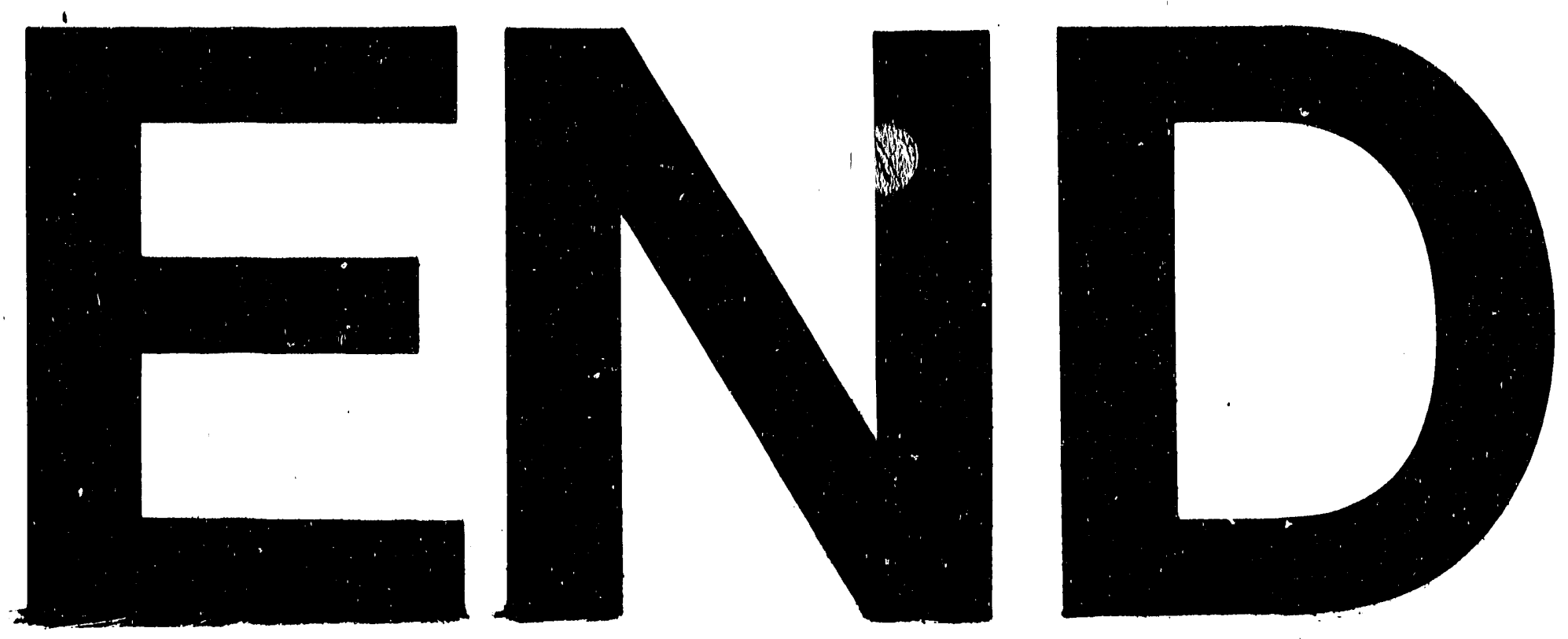

ANYI
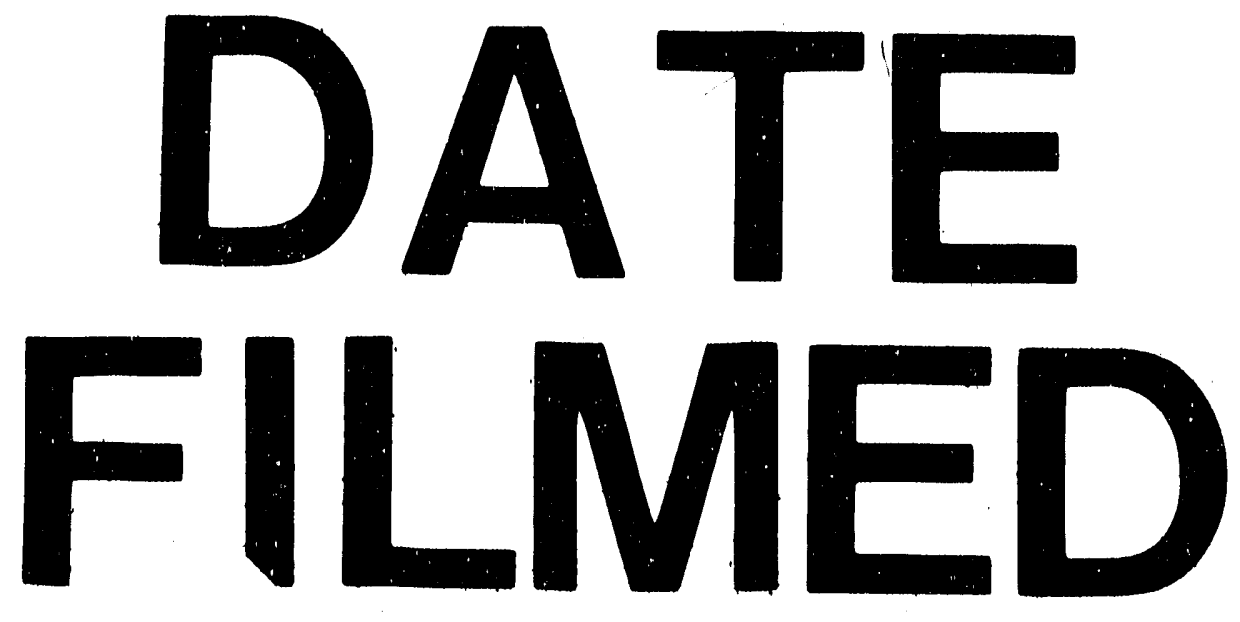

$6 / 22 / 92$ 
